\title{
Sexualisierte Gewalt gegen Kinder und Jugendliche mit Behinderungen: Erweiterter Forschungsstand seit 2014 und praktische Konsequenzen
}

\author{
Sexual Violence against Children and Adolescents with \\ Disabilities: Extended Research Status since 2014 and Practical \\ Implications
}

\author{
Autor:innen \\ Wencke Chodan ${ }^{1}$, Frank Häßler ${ }^{2}$ und Olaf Reis ${ }^{3}$ \\ Institute \\ 1 Fraunhofer IGD, Rostock \\ 2 Gesellschaft für Gesundheit und Pädagogik mbh, Rostock \\ 3 Klinik für Psychiatrie, Neurologie, Psychosomatik und \\ Psychotherapie im Kindes- und Jugendalter, Universitäts- \\ medizin Rostock
}

\section{Schlüsselwörter}

Beeinträchtigungen; Behinderung; Kindesmissbrauch;

Prävention; sexueller Missbrauch

Keywords

child sexual abuse; disability; impairments; prevention;

sexual abuse

Bibliografie

Z Sexualforsch 2021; 34: 137-151

DOI 10.1055/a-1553-0435

ISSN 0932-8114

(c) 2021. Thieme. All rights reserved.

Georg Thieme Verlag KG, Rüdigerstraße 14,

70469 Stuttgart, Germany

\section{Korrespondenzadresse}

Dr. rer. hum. Wencke Chodan

Fraunhofer Gesellschaft

Institut für Graphische Datenverarbeitung

Joachim-Jungius-Str. 11

18059 Rostock

Deutschland

wencke.chodan@igd-r.fraunhofer.de

\section{ZUSAMMENFASSUNG}

Einleitung Kinder und Jugendliche mit Behinderungen stellen eine Hochrisikogruppe dar, die signifikant häufiger von sexualisierter Gewalt betroffen ist als Kinder und Jugendliche ohne Behinderungen.

Forschungsziele Das vorliegende narrative Review aktualisiert den Überblick über die internationale Forschungslage zu Prävalenz, Risikofaktoren und Interventionen von und bei sexualisierter Gewalt gegen Kinder und Jugendliche mit Behinderungen.
Methoden Durch systematische Recherchen in Pubmed, PsycINFO und verschiedenen Suchmaschinen wurden 39 Publikationen extrahiert, die seit 2014 das Themenfeld der sexualisierten Gewalt gegen Kinder und Jugendliche mit Behinderungen beleuchten und damit einen Reviewartikel von 2014 aktualisieren.

Ergebnisse Die eingeschlossenen 39 Artikel wurden in die Bereiche Epidemiologie (Prävalenz $n=17$, Risikofaktoren $n=5$, Folgen sexualisierter Gewalt $n=2$ ), Versorgung (Prävention $n=7$, Intervention $n=6)$ und Verschiedenes $(n=2)$ unterteilt. Eine aktuelle Metaanalyse unterstreicht das erhöhte Risiko für Kinder und Jugendliche mit Behinderungen im Vergleich zu Gleichaltrigen ohne Behinderungen, von sexualisierter Gewalt betroffen zu werden. Zwei neu entwickelte, strukturierte und evaluierte Präventionsprogramme treten diesem Risiko entgegen. Für die Versorgung von Kindern und Jugendlichen nach dem Erleben von sexualisierter Gewalt gibt es lediglich für Kinder und Jugendliche mit Hörbehinderungen eine Publikation mit konkreten Hinweisen; für alle anderen Behinderungsformen ließ sich auch seit 2014 kein evaluiertes Programm auffinden. Schlussfolgerung Implikationen für künftige Forschung zu sexualisierter Gewalt gegen Kinder und Jugendliche mit Behinderungen und deren Versorgung werden diskutiert.

\section{ABSTRACT}

Introduction Children and adolescents with disabilities experience sexual violence significantly more often than children and adolescents without disabilities.

Objectives This narrative review updates the overview of the international research situation on the prevalence, risk factors and interventions regarding the sexual violence against children and adolescents with disabilities.

Methods By means of a systematic search in Pubmed, PsycINFO and various search engines, 39 publications were extracted that have shed light on the topic of sexual violence against children and adolescents with disabilities since 2014 and thus update a review article from 2014.

Results The 39 articles comprise the areas of epidemiology (prevalence $n=17$, risk factors $n=5$, and sequelae of abuse $n=2$ ), care (prevention $n=7$, intervention $n=6$ ), and miscellaneous 
$(n=2)$. A current meta-analysis underlines the increased risk of children and young people with disabilities experiencing sexual violence compared to their peers without disabilities. Two newly developed, structured and evaluated prevention programs counter this risk. Regarding the care of children and adolescents that have experienced sexual violence, there is only one publication with specific information that is for children and adolescents with hearing impairments; for all other forms of disability, no evaluated program could be found, including since 2014.

Conclusion Implications for future research on abuse and abuse prevention in children and adolescents with disabilities are discussed.
Das vorliegende narrative Review betrachtet den Forschungsstand zum Thema der sexualisierten Gewalt gegen Kinder und Jugendliche mit Behinderungen anhand von Studien, die dieses Phänomen quantitativ untersuchen und in Peer-Review-Fachzeitschriften publiziert wurden. Die Begriffe sexualisierte Gewalt und Behinderungen wurden gewählt, um innerhalb des Artikels eine einheitliche Nomenklatur zu schaffen. Der Begriff Behinderung in diesem Artikel meint Eigenschaften einer Person, die durch das aktuelle gesellschaftliche Umfeld zu Einschränkungen der Teilhabe führen und die international klassifiziert sind. Darunter fallen körperliche Eigenschaften, z. B. solche, die durch die Nutzung eines Rollstuhls ausgeglichen werden können, sensorische Eigenschaften wie Gehörlosigkeit, Blindheit, Schwerhörigkeit und Taubblindheit, kognitive Eigenschaften wie Intelligenzminderung, Eigenschaften der Sprache und des Sprechens sowie tiefgreifende psychische Eigenschaften wie Autismus (vgl. WHO 2007). Der Begriff der sexualisierten Gewalt meint in diesem Artikel jede sexualisierte Handlung, die unter bewusster Ausnutzung der Ungleichheit von Erfahrung, Wissen, Macht und Autorität vorgenommen wird (in Anlehnung an Hallstein 1996, zit. n. Häßler und Fegert 2005).

Kinder und Jugendliche mit Behinderungen gehören zu den Hochrisikogruppen im Hinblick auf sexualisierte Gewalt (Mailhot Amborski et al. 2021). Dennoch gibt es erhebliche Lücken im Verständnis und der wissenschaftlichen Untersuchung dieses Bereichs und insbesondere in den Angeboten an Präventions- und Interventionsprogrammen (vgl. Kendall-Tacket et. al 2005). Zudem bestehen weit verbreitete (und teils widersprüchliche) Vorurteile, die sexualisierte Gewalt begünstigen und eine Aufdeckung verhindern und die Brill (1998: 164) treffend zusammenfasst:

„So heißt es, Behinderte seien sexuell nicht attraktiv (für andere); ferner seien sie überhaupt asexuelle Wesen und hätten keinerlei Sexualität; sie könnten deshalb Missbrauch nicht verstehen; oder aber, sie seien promiskuitiv [...]; oder sexuelle Gewalt sei gar als eine Art Gefallen aufzufassen, da sie sonst überhaupt keine Sexualität erfahren könnten; und schließlich - zur Exkulpierung der Tat - sei die sexuelle ,Anmache“ von [der betroffenen Person] ausgegangen [...].“

Derartige Fehleinschätzungen führen dazu, dass die potenzielle Gefährdung durch sexualisierte Gewalt gegen Menschen mit Behinderungen weder durch die Gesellschaft noch durch einzelne Betreuende oder Sorgeberechtigte ausreichend wahrgenommen wird und Vorfälle nicht aufgedeckt werden. Bereits in den 1990erJahren wiesen viele Autor:innen und Studien darauf hin, dass Betroffene weniger geschützt werden beziehungsweise ihnen nicht beigestanden wird (Hames 1996; Mansell et al. 1992; Sobsey und Mansell 1994; Tharinger et al. 1990). Seligmann prägte 1996 daher den Ausdruck „Tabu im Tabu“.

Der vorliegende Artikel verfolgt daher das Ziel, die Ergebnisse bisheriger Forschung zu sexualisierter Gewalt gegen Kinder und Jugendliche mit Behinderungen zusammenzutragen und dabei ein früheres Forschungsreview (Chodan et al. 2014a) zu aktualisieren.

\section{Suchstrategie}

Die Suche erfolgte im April 2021 in den Datenbanken PubMed und PsycINFO sowie mithilfe der Suchmaschinen MetaGer, Google bzw. Google Scholar.

Als Suchwörter dienten:

- Suchwörter, die sich auf den Altersbereich beziehen (child* AND/OR adolescen*),

- gepaart mit Suchwörtern, die sich auf die Behinderungen beziehen (disab* OR impairment OR challenge* OR deficien* OR handicap OR limit* OR difficulties OR low functioning OR intellect* OR mental* OR retard*),

- gepaart mit Suchwörtern, die sich auf die sexualisierte Gewalt beziehen (sexual AND abuse OR violence OR offense OR maltreatment OR exploitation).

Die Suche wurde auf den Zeitraum von 2014 bis heute begrenzt, da sie eine Suche ergänzt, die initial 2011 erfolgte und zuletzt 2014 umfassend aktualisiert wurde (Chodan et al. 2014a). Berücksichtigt wurden englisch- und deutschsprachige Quellen.

Die Suche mit o.g. Kriterien ergab über alle Datenbanken mehrere tausend Treffer. Anhand der Titel wurden diejenigen aussortiert, die keinen Bezug zum Thema hatten und bei denen es sich nicht um Peer-Review-Publikationen von Studien oder Reviews handelte, die ihre Aussagen evidenzbasiert treffen. Es wurden $108 \mathrm{Ar}$ tikel ausgewählt, um deren Relevanz anhand der Abstracts gemäß folgender Einschlusskriterien zu bewerten:

1. Die Artikel mussten sich auf Kinder und Jugendliche beziehen (Ausschluss von neun Publikationen),

2. sich speziell mit Behinderungen befassen (Ausschluss von 39 Studien) und

3. sich speziell auf sexualisierte Gewalt beziehen (Ausschluss von elf Studien zu anderen Formen des Missbrauchs und zehn Studien zu Sexualität und sexueller Aufklärung bei Kindern und Jugendlichen mit Behinderungen).

Die eingeschlossenen 39 Artikel wurden nach ihrem Fokus in die Bereiche Epidemiologie (Prävalenz $n=17$, Risikofaktoren $n=5$, Fol- 
gen sexualisierter Gewalt $n=2$ ) und Versorgung (Prävention $n=7$, Intervention $n=6$ ) unterteilt. Die zwei verbleibenden Artikel befassen sich mit übergeordneten Themen (ein übergeordneter Reviewartikel von Wissink et al. von 2015 und ein Artikel zu ethischen, methodologischen und praktischen Herausforderungen bei der Erforschung von sexualisierter Gewalt gegen Kinder und Jugendliche mit Behinderungen von Fry et al. von 2017).

\section{Epidemiologie sexualisierter Gewalt gegen Kinder und Jugendliche mit verschiedenen Behinderungsformen}

\section{Prävalenz}

Das Risiko für Kinder und Jugendliche mit Behinderung, von sexualisierter Gewalt betroffen zu werden, ist größer ist als jenes für Kinder und Jugendliche ohne Behinderungen (Mailhot Amborski et al. 2021). Die größten Einzelstudien sind die von Sullivan und Knutson (2000) an über 40000 Kindern und Jugendlichen (davon $8.15 \%$ mit Behinderungen) und eine Publikation von 2017, die bevölkerungsbasiert alle westaustralischen Kinder und Jugendlichen, die zwischen 1990 und 2010 geboren wurden $(n \approx 525000$, davon $10.4 \%$ mit Behinderungen), auf Aktenlage untersucht (Maclean et al. 2017). Keine andere der aktuelleren Einzelstudien verfügt über eine vergleichbare Stichprobengröße (z. B. Dion et al. 2018; vgl. Wissink et al. 2015). Nur Euser et al. (2016) kommen über die befragten 104 Fachkräfte indirekt immerhin auf eine Stichprobengröße von 1650 Kindern und Jugendlichen, allerdings wurden hier ausschließlich Kinder und Jugendliche mit einer Intelligenzminderung in Fremdunterbringung betrachtet - im Gegensatz zu Sullivan und Knutson (2000), die einen breiteren Ansatz verfolgen und verschiedene Behinderungs- und Misshandlungsformen differenzieren und vergleichen. Auch andere der aufgefundenen Studien setzen ihren Schwerpunkt auf einzelne Behinderungsformen, z. B. Körperbehinderungen (Mueller-Johnson et al. 2014), Hörbehinderungen (Schenkel et al. 2014), neurologische Entwicklungsstörungen (Ohlsson Gotby et al. 2018), Autismus und Intelligenzminderung (McDonnell et al. 2019) oder Lernbehinderung (Helton et al. 2018). Wieder andere Studien fokussieren nur auf einen konkreten Ausschnitt sexualisierter Gewalt, z. B. Sex Trafficking gemäß Bundesstrafrecht der USA (Reid 2018) oder Pornografie (Henry et al. 2019), auf nur einen Teil von Kindern und Jugendlichen mit Behinderungen, z. B. auf fremduntergebrachte (Wissink et al. 2018), oder auf Teilaspekte der Prävalenz wie z. B. Unterberichterstattung (Willott et al. 2020) oder die Prävalenz aus der Wahrnehmung bestimmter Personengruppen wie Sonderschullehrer:innen (Büyükbayraktar et al. 2018; Kucuk et al. 2017).

Für ein umfassendes Review zur Prävalenz von sexualisierter Gewalt gegen Menschen mit Behinderungen sei auf Byrne (2018) verwiesen, der ein Review von Horner-Johnson und Drum (2006) aktualisiert. Ein weiteres Review zu dieser Thematik erschien von Corr und Santos (2017). Seit diesem Jahr liegt nun auch eine Metanalyse vor (Mailhot Amborski et al. 2021), die 68 englisch- und französischsprachige Einzelstudien aus 14 Ländern (vorwiegend aus den USA und England/UK, daneben aus Kanada, Australien, Norwegen, Italien, Deutschland, Niederlande, Israel, Taiwan, Nepal, Sri Lanka, Südafrika und Ghana) einschließt und damit 12427 Teilneh- mer:innen mit Behinderungen über 84 unabhängige Stichproben. 30 von diesen Studien beinhalten Minderjährige (definiert als Teilnehmer:innen unter 21 Jahren). Die Ergebnisse dieser Metaanalyse zeigen mittels Odds Ratio (OR), dass Personen mit Behinderungen ein über zweifach höheres Risiko haben, in ihrem Leben von sexualisierter Gewalt betroffen zu werden, als Menschen ohne Behinderung $(O R=2.27)$. Für die Studien mit Minderjährigen ergibt sich ein OR von 1.40, wobei diese Zahl lediglich die erhöhte Vulnerabilität von Kindern und Jugendlichen mit Behinderungen im Vergleich zu Kindern und Jugendlichen ohne Behinderungen aufzeigt und sich nicht mit den 2.27 ins Verhältnis setzen lässt (aufgrund der unterschiedlich großen Zeiträume). Im Gegenteil berichtet bereits ein alter Reviewartikel von 1991, dass die Gefährdung von Menschen mit Behinderung besonders hoch sei, wenn sie jung sind (Baladerian 1991). Nach Baladerian (1991) erleben 39-83\% der Frauen und 16-37\% der Männer mit Intelligenzminderung vor ihrem 18. Geburtstag sexuelle Übergriffe.

Nach der Studie von Sullivan und Knutson (2000), die in der Metanalyse enthalten ist, sind Kinder und Jugendliche mit einer Behinderung etwa dreimal so häufig von Misshandlungen betroffen ( $31 \%, n=3262)$ wie Kinder und Jugendliche ohne Behinderungen ( $9 \%, n=36949)$. Unter Misshandlung werden hierbei neben sexualisierter Gewalt auch körperliche Misshandlung, emotionaler Missbrauch sowie Vernachlässigung betrachtet. Die Studie unterscheidet zudem zwischen verschiedenen Behinderungsformen. Bezogen auf sexualisierte Gewalt sind Kinder und Jugendliche mit Verhaltensstörungen davon am häufigsten betroffen (Relatives Risiko RR = 5.5), gefolgt von Kindern und Jugendlichen mit Intelligenzminderung $(R R=4.0)$ und Kindern und Jugendlichen mit Beeinträchtigungen des Sprechens und der Sprache $(R R=2.9)$. Auch Kinder und Jugendliche mit Körperbehinderungen ( $R R=2.0)$, gesundheitsbezogenen Behinderungen ( $R R=2.0)$ bzw. sensorischen Behinderungen (Seh- bzw. Hörbehinderungen) sowie Kinder und Jugendliche mit einer Lernbehinderung $(R R=1.8)$ sind in dieser Studie gemäß ihren Angaben häufiger von sexualisierter Gewalt betroffen als Kinder und Jugendliche ohne Behinderungen. Lediglich für Kinder und Jugendliche mit Autismus ergibt sich kein signifikant erhöhtes Relatives Risiko, was jedoch auch auf die geringe Stichprobengröße dieser Gruppe zurückzuführen sein könnte.

Auch die Metanalyse (Mailhot Amborski et al. 2021) bestätigt, dass die Art der Behinderung einen signifikanten moderierenden Effekt hat, wobei hier leider nicht nach dem Alter unterschieden wird, sodass Studien zu Erwachsenen und zu Kindern und Jugendlichen gemeinsam betrachtet werden. Das höchste Risiko, sexualisierte Gewalt zu erleben, besteht demnach in der Gruppe der Menschen mit sensorischen Behinderungen ( $O R=7.57$, Anzahl der Studien $k=12$ ), gefolgt von den anderen Formen von Behinderungen (Intelligenzminderung: $\mathrm{OR}=1.81, \mathrm{k}=24$; körperliche Behinderung: $\mathrm{OR}=1.71, \mathrm{k}=16$, gemischte Arten von Behinderungen: $\mathrm{OR}=1.76$, $\mathrm{k}=28$ ). Autismus und Sprachbeeinträchtigung sind nicht berücksichtigt worden, da die Anzahl der Studien $(k<4)$ nicht ausreichend war.

Neben der Form der Behinderung ist das Geschlecht ein weiterer Risikofaktor: Innerhalb der Gruppe der misshandelten Kinder und Jugendlichen mit Behinderung sind - ähnlich wie in der Gruppe der Kinder und Jugendlichen ohne Behinderungen - Mädchen signifi- 
- Tab. 1 Prävalenz von Misshandlungen von Kindern und Jugendlichen mit Behinderungen in Prozent (nach Sullivan und Knutson 2000), aufgeschlüsselt nach verschiedenen Behinderungsformen (Spalten) und Misshandlungsformen (Zeilen).

\begin{tabular}{|c|c|c|c|c|c|}
\hline $\begin{array}{lll}\text { Misshandlungsform } & \text { Behinderungsform }\end{array}$ & $\begin{array}{l}\text { Verhaltens- } \\
\text { störung }{ }^{1}\end{array}$ & $\begin{array}{l}\text { Kommunika- } \\
\text { tionsstörung }\end{array}$ & $\begin{array}{l}\text { Gesundheit/ } \\
\text { orthopädisch }^{3}\end{array}$ & $\begin{array}{l}\text { Geistige } \\
\text { Behinderung }\end{array}$ & Gesamt \\
\hline Vernachlässigung & 26.2 & 21.9 & 23.0 & 21.8 & 23.6 \\
\hline Sexuell & 3.0 & 8.9 & 6.3 & 8.5 & 6.2 \\
\hline Physisch & 5.4 & 8.4 & 6.3 & 4.8 & 6.1 \\
\hline Emotional & 0.3 & 0.4 & 1.6 & 0.4 & 0.5 \\
\hline \multicolumn{6}{|l|}{ Kombination von Misshandlungsformen } \\
\hline Sexuell/physisch & 1.4 & 1.3 & 1.6 & 2.0 & 1.5 \\
\hline Physisch/Vernachlässigung & 11.4 & 12.7 & 9.5 & 11.3 & 11.5 \\
\hline Sexuell/emotional & 0.0 & 0.4 & 0.0 & 0.0 & 0.1 \\
\hline Physisch/emotional & 1.6 & 0.8 & 2.4 & 0.4 & 1.2 \\
\hline Sexuell/Vernachlässigung & 3.3 & 2.5 & 4.8 & 6.0 & 4.0 \\
\hline Emotional/Vernachlässigung & 8.2 & 15.6 & 15.1 & 9.3 & 11.1 \\
\hline Sexuell/physisch/emotional & 0.8 & 0.0 & 0.0 & 0.0 & 0.3 \\
\hline Physisch/Vernachlässigung/emotional & 15.5 & 13.9 & 17.5 & 15.3 & 15.3 \\
\hline Sexuell/Vernachlässigung/emotional & 4.1 & 2.5 & 3.2 & 4.0 & 3.6 \\
\hline Sexuell/physisch/Vernachlässigung & 4.6 & 3.0 & 5.6 & 4.4 & 4.3 \\
\hline Sexuell/physisch/emotional/Vernachlässigung & 11.2 & 7.2 & 2.4 & 11.7 & 9.2 \\
\hline Keine Angaben & 3.0 & 0.4 & 0.8 & 0.0 & 1.3 \\
\hline Gesamt & $\begin{array}{l}100.0 \\
(n=367)\end{array}$ & $\begin{array}{l}100.0 \\
(n=237)\end{array}$ & $\begin{array}{l}100.0 \\
(n=126)\end{array}$ & $\begin{array}{l}100.0 \\
(n=248)\end{array}$ & $\begin{array}{l}100.0 \\
(n=978)\end{array}$ \\
\hline
\end{tabular}

Anm.: Für diese Auswertung wurden Formen von Behinderung zusammengefasst.

1 Die Verhaltensstörungen umfassen zusätzlich den Autismus.

2 Kommunikationsstörungen vereinen Sprach-, Hör- und Lernbehinderungen.

${ }^{3}$ Orthopädische und Gesundheitsbehinderungen umfassen neben Sehbehinderungen orthopädische Behinderungen und gesundheitsbezogene Behinderungen wie Asthma.

kant häufiger von sexualisierter Gewalt betroffen als Jungen (Sullivan und Knutson 2000; z. B. 80 \% weibliche Betroffene bei Mansell und Sobsey 1992).

-Tab. 1 unterteilt die einzelnen Misshandlungsformen, die neben sexualisierter Gewalt Vernachlässigung, physische Misshandlung, emotionale Misshandlung und Kombinationen von Misshandlungsformen unterscheiden. Insgesamt geben 29.2\% aller Kinder und Jugendlichen mit Behinderung an, bereits sexuelle Übergriffe erlebt zu haben (alleinstehend sowie in allen Kombinationen mit anderen Missbrauchsformen; Sullivan und Knutson 2000).

Eine nationale Umfrage des BMFSFJ (2013) mit 1561 Teilnehmerinnen stützt diese Ergebnisse zusätzlich. In der Umfrage geben 20-34\% der Frauen mit Behinderung und Beeinträchtigungen retrospektiv an, sexualisierte Gewalt in Kindheit und Jugend erlebt zu haben, wobei die Tatpersonen Erwachsene waren. Sie sind damit etwa zwei- bis dreimal häufiger davon betroffen als Frauen ohne Behinderungen (10\%). Wird zusätzlich auch sexualisierte Gewalt durch andere Kinder und Jugendliche betrachtet, dann hat je nach Untersuchungsgruppe jede zweite bis vierte Frau der Studie sexuelle Übergriffe in Kindheit und Jugend erlebt, allen voran gehörlose Frauen ( $52 \%$ ), gefolgt von blinden Frauen ( $40 \%$ ), psychisch erkrankten Frauen (36\%), körper-/mehrfachbehinderten Frauen (34\%) und Frauen mit einer Intelligenzminderung (25\%).

\section{Chronische sexualisierte Gewalt}

In 48-81\% der Fälle von sexualisierter Gewalt gegen Kinder und Jugendliche mit Behinderungen handelt es sich um mehrmalige Übergriffe, die über längere Zeit andauern (Mansell et al. 1992; McCarthy und Thompson 1997; vgl. Caldas und Bensy 2014). Auch gemäß der Studie des BMFSFJ (2013) müssen Frauen mit Behinderungen fortgesetzte und multiple Gewalterfahrungen in Kindheit und Jugend erheblich häufiger erleben als Frauen im Bevölkerungsdurchschnitt (z. B. 16\% bei Frauen mit Intelligenzminderung im Vergleich zu $7 \%$ bei Frauen ohne Behinderung).

\section{Tatpersonen}

Die Tatpersonen sind vorwiegend männlich $(90.8 \%$ ) und den Betroffenen in $91 \%$ der Fälle bekannt (Balogh et al. 2001; Mansell et al. 1992).

Etwa 10\% der sexuellen Übergriffe geschehen durch andere Kinder und Jugendliche (BMFSFJ 2013), darunter auch andere Kinder und Jugendliche mit Behinderungen (Mansell et al. 1992). Häufiger handelt es sich jedoch um erwachsene Tatpersonen, insbesondere Verwandte und Pflege- und Betreuungspersonal (26.3\% Betreuende, $6 \%$ Transportbereitstellende, $17 \%$ biologisch verwandte Familienmitglieder, 4.5\% Pflegefamilienmitglieder, 0.5\% Stiefgeschwister; Mansell et al., 1992). Damit sind die Tatpersonen und die sexualisierte Gewalt nicht nur im Nahbereich von Kindern und 
Jugendlichen mit Behinderungen angesiedelt, sondern mehr noch in Bereichen, die eigentlich eine Schutzfunktion für die Kinder und Jugendlichen innehaben.

Die Zahlen lassen sich nur schwer mit der Verteilung der Tatpersonen bei Betroffenen ohne Behinderungen vergleichen, da hier zum Teil die Unterteilung der außerfamiliären Tatpersonen in Bekannte aus dem sozialen Umfeld (z. B. Nachbarn, Freunde der Eltern) und Bekannte aus dem professionellem Umfeld (z. B. Betreuende, Tatpersonen aus Vereinen oder Institutionen) fehlt (z. B. bei der Statistik des Bundeskriminalamts; zit. n. Bange und Enders 2012) und/oder die Zahlen getrennt nach Geschlechtern aufgeführt werden, wobei es zwischen den Geschlechtern deutliche Unterschiede gibt. Dies ist beispielsweise der Fall bei der wissenschaftlichen Auswertung der telefonischen Meldungen und Briefe von Betroffenen der Unabhängigen Beauftragten zur Aufarbeitung des sexuellen Kindesmissbrauchs (Bange und Enders 2012). Hiernach erleben $70.8 \%$ der weiblichen Betroffenen, aber nur 32.6\% der männlichen Betroffenen sexualisierte Gewalt durch Verwandte - insgesamt ein höherer Anteil als in der Gruppe der Kinder und Jugendlichen mit Behinderungen. Eine Aufteilung in biologische Familien und Pflegefamilien erfolgt hierbei nicht. 17.2\% der weiblichen und $56.9 \%$ der männlichen Betroffenen erleben nach dieser Quelle sexualisierte Gewalt in Vereinen, Ferienbetreuung, Pfarrgemeinden und Institutionen, wobei hier keine Unterteilung in Freizeitgestaltung und notwendige Betreuung erfolgt (Bange und Enders 2012).

\section{Folgen}

Kinder und Jugendliche mit Behinderungen erleben häufiger als Kinder und Jugendliche ohne Behinderungen sexualisierte Gewalt im Nahbereich (z. B. durch Betreuungspersonal) und fortgesetzte sexualisierte Gewalt. Chronische sexualisierter Gewalt durch eine bekannte oder vertraute Person wirkt pathogener als ein einmaliger Vorfall sexualisierter Gewalt durch eine unbekannte Tatperson (Mansell und Sobsey 2001). Die Auswirkungen von sexualisierter Gewalt auf Kinder und Jugendliche mit Behinderungen sind symptomatisch ähnlich wie bei Gleichaltrigen ohne Behinderungen (Byrne 2018) oder können aufgrund begrenzter Bewältigungsressourcen schwerwiegender sein (Friedrich 1998; Mansell et al. 1992). Austin et al. (2016) untersuchen die psychiatrischen sowie psychosozialen Auswirkungen von sexualisierter Gewalt und Misshandlungen im Kindes- und Jugendalter an erwachsenen Personen. Personen mit Behinderungen zeigen dabei eine schlechtere körperliche und geistige Gesundheit (z. B. vom Arzt diagnostizierte Depression) als Personen ohne Behinderung. Nur eine sehr geringe Zahl der Betroffenen zeigt keinerlei Auswirkungen (z. B. zit. n. Mansell et al. 1992 oder Sobsey und Doe 1991; beide zit. n. Sequeira und Hollins 2003, wobei das Review auch methodische Mängel der Studien offenlegt).

\section{Spezifische Risikokonstellationen und Risikofaktoren}

Das Risiko, sexualisierte Gewalt zu erleben, wird durch gesellschaftlich oder institutionell künstlich geschaffene Bedingungen erhöht, z. B. dadurch, dass Kinder und Jugendliche mit Behinderungen nach wie vor häufiger sozial isoliert aufwachsen (Franklin und Smeaton 2017). Diese soziale Isolation kann zu einem starken Wunsch nach Zuneigung und Zuwendung führen, die Tatpersonen unter Umständen den Zugang zu den Betroffenen erleich- tert (Normand und Sallafranque-St-Louis 2016), und sie kann das Berichten erschweren.

Kinder und Jugendliche mit Behinderungen werden - mehr noch als Kinder und Jugendliche ohne Behinderungen - in vielen Lebensbereichen fremddominiert (Franklin und Smeaton 2017) und zur Compliance (Regelbefolgung) erzogen, sodass ihnen Abgrenzungen und Selbstbestimmung äußerst schwerfallen. Oft wissen sie weder, welche Rechte sie haben - auch auf ihre körperliche Selbstbestimmung bezogen -, noch können sie diese effektiv artikulieren. Gesellschaft, Familie und Institutionen sollten ein Umfeld schaffen, in dem die Kinder und Jugendlichen ein gesundes Selbstbewusstsein ausbilden können, welches sich vielfach als Schutzfaktor erwiesen hat (Cederblad et al. 1994), während eine geringe Selbstsicherheit, die aus einer gesellschaftlich gelebten Fremddominierung resultiert, als Risikofaktor gilt (Tharinger et al. 1990).

Ein spezifisches Risiko der Kinder und Jugendlichen mit Behinderung ist ihre (physische und emotionale) Abhängigkeit von potenziellen Tatpersonen (Furey et al. 1994; O’Day 1983). Die Abhängigkeitsverhältnisse bestimmen auch die Manipulationsversuche der Tatpersonen, die von Kindern und Jugendlichen mit Behinderungen seltener durchschaut werden, was neben der Abhängigkeit und sozialen Isolation auch auf das bestehende vertrauensvolle Verhältnis zu den Tatpersonen zurückzuführen ist (Noack und Schmid 1994; Senn 1993;) oder, je nach Form der Behinderung, auch auf ein begrenztes kognitives Verständnis, z. B. bei einer Intelligenzminderung.

Ein wichtiger Unterschied in der psychosexuellen Entwicklung zwischen Kindern und Jugendlichen mit Behinderungen und Kindern und Jugendlichen ohne Behinderungen besteht im gesellschaftlich mitbestimmten Erleben kritischer Entwicklungsphasen. Die Ungleichzeitigkeit zwischen körperlich-biologischer und psychosozial-kognitiver Entwicklung lässt ein großes Spannungspotenzial entstehen, welches die Betroffenen, aber auch die Sorgeberechtigten oder Betreuenden, häufig überfordert (Stinkes 2006). Kinder mit Behinderungen entwickeln ungefähr zum gleichen Zeitpunkt wie Heranwachsende ohne Behinderungen sexuelle Neigungen und körperliche Reife (Matson und Sevin 1988), haben jedoch aufgrund gesellschaftlicher Bedingungen geringere Chancen, ihre Sexualität zu verstehen und auszuprobieren (Tharinger et al. 1990; Vara et al. 2019). Damit erhöhen die wahrscheinlichen Asynchronien von körperlicher, kognitiver und sozioemotionaler Entwicklung das Risiko, von sexueller Gewalt betroffen zu werden.

Nach Tang und Lee (1999) ist das Wissen über sexualisierte Gewalt diejenige Variable, die am ehesten die Fähigkeit, selbstschützende Fertigkeiten zu mobilisieren, vorhersagt (unabhängig vom Alter und intellektuellen Funktionsniveau). Die fehlende oder mangelnde Sexualaufklärung, die Nichtthematisierung von Sexualität und sexuellen Übergriffen ist somit ein gewichtiger weiterer gesellschaftlicher Risikofaktor (Fenwick 1994; Ludlow 1991), der infolge der tabuisierten Sexualität besonders auf Kinder und Jugendliche mit Behinderungen zutrifft. Die unzureichende Differenzierung von normalen vs. übergriffigen Situationen erschwert deren Erkennen, Benennen und Berichten. Wer nicht über ein differenziertes Konzept von Intimität und Körperwissen verfügt, hat Probleme in der Definition von Grenzüberschreitungen und kann sich kaum angemessen wehren oder darüber sprechen (vgl. Stinkes 2006). Indirekt erschwert so eine mangelnde Sexualaufklärung die Aus- 
- Tab.2 Risikofaktoren für sexuellen Missbrauch und die Studien, die diese für Kinder und Jugendliche mit Behinderungen jeweils identifiziert haben.

\begin{tabular}{|c|c|}
\hline Risikofaktor & Studien \\
\hline Soziale Isolation & Franklin und Smeaton 2017; Normand und Sallafranque-St-Louis 2016 \\
\hline Fremddominierung, Erziehung zur Compliance (Regelbefolgung) & $\begin{array}{l}\text { Bruder und Stenfert Kroese 2005; Franklin und Smeaton 2017; Lumley } \\
\text { und Miltenberger 1997; Muccigrosso 1991; Tang und Lee 1999; } \\
\text { Tharinger et al. } 1990\end{array}$ \\
\hline $\begin{array}{l}\text { (physische und emotionale) Verbundenheit zu oder Abhängigkeit von } \\
\text { potenziellen Tatpersonen }\end{array}$ & Furey et al. 1994; Noack und Schmid 1994; O’Day 1983; Senn 1993 \\
\hline $\begin{array}{l}\text { Riskante Betreuungsstrukturen in Familien und institutionelle Risiken, } \\
\text { wie z. B. Isolation oder ein fehlendes oder zu komplexes Beschwerde- } \\
\text { management }\end{array}$ & $\begin{array}{l}\text { Bruder und Stenfert Kroese 2005; Lumley und Miltenberger 1997; } \\
\text { Muccigrosso 1991; Tang und Lee 1999; Tharinger et al. } 1990\end{array}$ \\
\hline Mangelnde Aufdeckung des Missbrauchs & $\begin{array}{l}\text { Bruder und Stenfert Kroese 2005; Lumley und Miltenberger 1997; } \\
\text { Muccigrosso 1991; Tang und Lee 1999; Tharinger et al. } 1990\end{array}$ \\
\hline $\begin{array}{l}\text { Abwertende und stigmatisierende kulturelle, gesellschaftliche und } \\
\text { familiäre Einstellungen gegenüber Menschen mit Behinderungen }\end{array}$ & $\begin{array}{l}\text { Belsky 1980; Bronfenbrenner 1977; Garbarino und Stocking 1980; } \\
\text { Ludlow } 1991\end{array}$ \\
\hline Geringe Akzeptanz des eigenen Körpers & Bruder und Stenfert Kroese 2005; Tharinger et al. 1990 \\
\hline Geringe Selbstsicherheit/geringes Selbstbewusstsein & Tharinger et al. 1990; vgl. Cederblad et al. 1994 \\
\hline $\begin{array}{l}\text { Tabuisierung ihrer Sexualität; geringere Chancen, die eigene Sexualität } \\
\text { zu verstehen und auszuprobieren; Unterdrückung von Sexualität in } \\
\text { Institutionen }\end{array}$ & Brill 1998; Hames 1996; Vara et al. 2019 \\
\hline $\begin{array}{l}\text { Fehlende oder mangelhafte Sexualaufklärung, fehlendes Wissen über } \\
\text { sexuellen Missbrauch }\end{array}$ & Fenwick 1994; Ludlow 1991; Stinkes 2006; Tang und Lee 1999 \\
\hline $\begin{array}{l}\text { Asynchronie (Ungleichzeitigkeit) von körperlicher, kognitiver und } \\
\text { sozioemotionaler Entwicklung }\end{array}$ & Matson und Sevin 1988; Stinkes 2006; Tharinger et al. 1990 \\
\hline Wunsch nach Zuneigung und Zuwendung & $\begin{array}{l}\text { Bruder und Stenfert Kroese 2005; Lumley und Miltenberger 1997; } \\
\text { Muccigrosso 1991; Tang und Lee 1999; Tharinger et al. 1990; Vara et al. } \\
2019\end{array}$ \\
\hline Unangemessene soziale Fertigkeiten & Singer 1996; Sobsey und Mansell 1994 \\
\hline Geringes Urteilsvermögen; Schwierigkeiten bei Entscheidungsprozessen & $\begin{array}{l}\text { Bruder und Stenfert Kroese 2005; Lumley und Miltenberger 1997; } \\
\text { Muccigrosso 1991; Tang und Lee 1999; Tharinger et al. } 1990\end{array}$ \\
\hline Mangel an Bewältigungsstrategien und Emotionsregulation & $\begin{array}{l}\text { Bruder und Stenfert Kroese 2005; Lumley und Miltenberger 1997; } \\
\text { Muccigrosso 1991; Tang und Lee 1999; Tharinger et al. } 1990\end{array}$ \\
\hline Mangel an Wissen über präventive Abwehrstrategien & Haseltine und Miltenberger 1990; Watson et al. 1992 \\
\hline Mangel an präventiven (selbstschützenden) Fertigkeiten & $\begin{array}{l}\text { Haseltine und Miltenberger 1990; Tang und Lee 1999; Watson et al. } \\
1992\end{array}$ \\
\hline $\begin{array}{l}\text { Probleme beim Berichten von Vorfällen (Vokabular, Gedächtnis- } \\
\text { speicherung und -abruf), eingeschränkte Kommunikationsfähigkeit }\end{array}$ & $\begin{array}{l}\text { Becker 1995; Bruder und Stenfert Kroese 2005; Lumley und Miltenberger } \\
\text { 1997; Muccigrosso 1991; Singer 1996; Sobsey und Mansell 1994; Tang } \\
\text { und Lee 1999; Tharinger et al. } 1990\end{array}$ \\
\hline Geringe zugeschriebene Glaubwürdigkeit & $\begin{array}{l}\text { Bruder und Stenfert Kroese 2005; Lumley und Miltenberger 1997; } \\
\text { Muccigrosso 1991; Tang und Lee 1999; Tharinger et al. } 1990\end{array}$ \\
\hline Sozioökonomische Risiken, z. B. Armut oder Arbeitslosigkeit in der Familie & Araten-Bergman et al. 2017 \\
\hline
\end{tabular}

bildung eines angemessenen Verhältnisses zum eigenen Körper: Die Kinder - insbesondere Kinder mit körperlichen Behinderungen - lernen nicht, dass ihr Körper etwas Schönes, Kostbares und Schützenswertes ist. Eine geringe Akzeptanz des eigenen Körpers wird hier als weiterer Risikofaktor herausgestellt (vgl. Bruder und Stenfert Kroese 2005; Tharinger et al. 1990).

Diese und weitere Risikofaktoren (z. B. sozioökonomische Risikofaktoren; vgl. Araten-Bergman et al. 2017 oder eine eingeschränkte Kommunikationsfähigkeit; vgl. Becker 1995), die in Situationen sexualisierter Gewalt gegen Kinder und Jugendliche mit Behinderungen kumulieren, sind in $>$ Tab. 2 zusammengefasst. Zwei weitere aktuelle Publikationen, die Risikokonstellationen betrachten, tun dies allgemein für Kinder und Jugendliche, sodass hier zwar Behinderungen als ein Risikofaktor herausgestellt werden, aber innerhalb der Gruppe von Kindern und Jugendlichen mit Behinderungen keine feinere Unterteilung erfolgt (Meinck et al. 2015a, 2015b).

\section{Prävention, Intervention und rechtliche Verfolgung}

\section{Prävention von sexualisierter Gewalt gegen Kinder und Jugendliche mit Behinderungen}

Die Prävention sexualisierter Gewalt ist mehrdimensional (Brown et al. 1994) und umfasst verschiedene, ineinandergreifende Ansätze. Zum einen sollen den potenziellen Betroffenen Selbstsicherheit und Fertigkeiten zum Selbstschutz beigebracht werden (Betroffenenperspektive). Um Kinder und Jugendliche mit Behinderungen 
selbst zu stärken (Empowerment) und sie in die Lage zu versetzen, sexualisierte Gewalt zu erkennen und zu beenden (wozu auch das Berichten gegenüber einer erwachsenen Vertrauensperson zählt), kommen verschiedene Trainings zur Anwendung, von denen nur wenige wissenschaftlich evaluiert sind (vgl. Chodan et al. 2014a) und die alleinig keinen ausreichenden Schutz bieten, wie für Kinder und Jugendliche mit Intelligenzminderungen gezeigt wurde (Reis et al., eingereicht zur Publikation).

Dazu sollen potenzielle Tatpersonen vom Kontakt mit Kindern und Jugendlichen mit Behinderung ferngehalten und behandelt werden (Tatpersonenperspektive). Insbesondere in den betreuenden Institutionen sollen Verhaltensrichtlinien für Mitarbeitende entwickelt werden (institutionelle Perspektive). Sexualisierte Gewalt soll aktiv aufgedeckt und gestoppt werden (gesellschaftliche Perspektive). Präventive Maßnahmen umfassen damit z. B. klare institutionelle Regeln, ein kindgerechtes sowie behindertengerechtes Beschwerdemanagement, die Verfügbarkeit sowohl interner als auch externer Ansprechpersonen (z. B. Missbrauchsbeauftragte), regelmäßige Präventionsangebote für Erwachsene (z. B. Sorgeberechtigte, Lehrkräfte, Betreuende), eine sorgfältige Personalauswahl (z. B. Einholen des erweiterten Führungszeugnisses) und einen verbindlichen Verhaltenskodex für die Angestellten (Bange 2014).

In der Aktualisierung konnten sieben Publikationen identifiziert werden, die sich mit der Prävention von sexualisierter Gewalt gegen Kinder und Jugendliche beschäftigen, darunter ein Review in einem Buchkapitel (Stone 2018), ein Studienprotokoll (Chodan et al. 2017) und eine unsystematische Beschreibung einer Fallvignette (Brisma und Amir 2020). Die restlichen vier Publikationen (Kim 2016; Thomas at al. 2018; Urbann et al. 2020; Warraitch et al. 2021) sind Originalarbeiten, die über Messwiederholungsdesigns die Effektivität eingesetzter Präventionsprogramme untersucht haben.

Die nun folgende Analyse der Publikationen und der Präventionslandschaft geschieht getrennt nach der Art der Behinderung, da Präventionsangebote auf diese zugeschnitten sein müssen. Zunächst wird die Studienlage zu Kindern und Jugendlichen mit einer Intelligenzminderung beschrieben; anschließend folgt die Analyse der Versorgungssituation für Kinder und Jugendliche mit Körperbehinderungen.

Bezogen auf Kinder und Jugendliche mit einer Intelligenzminderung existieren international sieben (englisch- oder deutschsprachige) in Fachzeitschriften publizierte Studien. Davon zählen drei zu den oben genannten Originalarbeiten, die in der Aktualisierung aufgefunden wurden (Kim 2016; Thomas et al. 2018; Warraitch et al. 2021). Die restlichen vier Studienpublikationen sind älter und wurden bereits in Chodan et al. 2014a ausführlich beschrieben und kritisch diskutiert (Andrews 1996, zit. n. Bruder und Stenfert Kroese 2005; Lee et al. 2001; Lee und Tang 1998; Llewellyn und McLaughlin 1987).

In Kim (2016) durchliefen drei Teilnehmerinnen im Alter von 11-13 Jahren mit leichter bis mittelgradiger Intelligenzminderung ein Behavioral Skill Training (BST). Die Erhebung der Wirksamkeit erfolgte mit möglichst reliablen (ebenfalls verhaltensnahen) Methoden (in situ Tests; vgl. Chodan et al. 2014a und Lumley und Miltenberger 1997). Im Vergleich zum Vortest verfügten die Teilnehmerinnen nach dem Training über bessere präventive Fertigkeiten, die sie zum Follow-up (zehn Wochen später) erhalten konnten. Die Aussagefähig- keit der Studie ist durch die geringe Stichprobengröße und das Fehlen einer Kontrollgruppe jedoch erheblich eingeschränkt.

In Thomas et al. (2018) wurden 14 Teilnehmer:innen (acht Jungen und sechs Mädchen im Alter von 10-18 Jahren) mit einer leichten oder mittelgradigen Intelligenzminderung jeweils hälftig in eine Experimentalgruppe und eine Kontrollgruppe aufgeteilt, wobei die Kontrollgruppe keine Intervention erhielt. Als abhängige Variablen wurden das Präventionswissen über den „Personal Safety Questionnaire“ (PSQ) und das imaginierte Präventionsverhalten (kein reales oder beobachtbares Verhalten) in einem videobasierten WhatIf-Situations-Test (WIST) abgefragt, wobei die Experimentalgruppe nach zwölf Sitzungen Behavioral Skill Training (BST), verteilt über einen Monat, in beiden Tests signifikant besser abschnitt als zuvor sowie besser als die Kontrollgruppe. Die Aussagefähigkeit der Studie ist durch die geringe Stichprobengröße und das Fehlen valider (verhaltensnaher und realitätsnaher) Methoden (vgl. Lumley und Miltenberger 1997) eingeschränkt.

In Warraitch et al. (2021) nahmen 15 Schülerinnen mit einer leichten Intelligenzminderung an einer Pilotstudie teil, die die Machbarkeit, die Akzeptanz und den Effekt eines fünf Sitzungen langen Präventionsprogramms mit positivem Ergebnis testete. Die Aussagefähigkeit der Studie ist durch die geringe Stichprobengröße, das Fehlen einer Kontrollgruppe und das Fehlen valider (verhaltensnaher und realitätsnaher) Methoden (vgl. Lumley und Miltenberger 1997) eingeschränkt.

Prinzipiell berichten alle Studien über positive Ergebnisse der Präventionsprogramme (vgl. Chodan et al. 2014a; z. B. vermehrtes Anwenden von präventiven Verhaltensweisen), mit nur einer Ausnahme (Andrews 1996, zit. n. Bruder und Stenfert Kroese 2005). Die Methodiken der Studien weisen jedoch teils gravierende Mängel auf, darunter geringe Stichprobengrößen, unstimmige Eingangskriterien (z. B. IQ bis in den Normbereich hinein trotz des Fokus auf Intelligenzminderung) oder mangelnde Validität der Erhebungsmethode der Befragung (vgl. Lumley et al. 1998). Trotz der methodischen Mängel ist dies ein Hinweis darauf, dass Präventionsmaßnahmen beziehungsweise Trainings mit potenziellen Betroffenen auch in dieser speziellen Population effektiv sind. Ein Manual liegt für keines der Trainingsprogramme vor.

Das Studienprotokoll von Chodan et al. (2017) beschreibt eine weitere Studie, die seit 2014 durchgeführt wurde. Sie ist Teil eines durch das BMBF geförderten Projektclusters, in dem in Deutschland von 2012 bis 2016 zwei hochstrukturierte und standardisierte Präventionstrainings für Kinder und Jugendliche mit Behinderungen entwickelt und aufwändig evaluiert wurden (eines für Kinder und Jugendliche mit Intelligenzminderungen und eines zu Kindern und Jugendlichen mit Hörbehinderungen, s. u.).

Chodan et al. (2017) beschreiben die Entwicklung und Evaluation eines Präventionsprogramms gegen sexualisierte Gewalt für Mädchen mit Intelligenzminderung (namens „Emma unantastbar! “1 ). Das Präventionstraining liegt als Gruppentraining für Mädchen mit Intelligenzminderung (8-12 Jahre) vor. Es umfasst zehn Sitzungen, in denen die Teilnehmerinnen anhand konkreter Beispiele in Rollenspielen und anderen kindgerechten und videounterstützten Übungen die Verhaltenskette „Nicht mitmachen - Nein sagen - Situation beenden - Situation berichten“ lernen, um sie so-

1 https://emma-unantastbar.med.uni-rostock.de/ 
wohl kurzfristig als auch langfristig vor weiteren sexuellen Übergriffen zu schützen. Das Trainingsprogramm wurde an über $100 \mathrm{Kin}-$ dern evaluiert, indem das Wissen, das imaginierte Verhalten und auch das tatsächlich gezeigte Verhalten in Rollenspielen und in situ Tests gemessen wurde (Chodan et al. 2017). Die Evaluation des Programmes soll demnächst erscheinen (Reis et al., eingereicht zur Publikation) und die Veröffentlichung des Manuals ist in Arbeit (Informationen bei der Erstautorin).

Die vierte seit 2014 publizierte Studie zur Prävention von sexualisierter Gewalt (Urbann et al. 2020) behandelt die Prävention für Kinder und Jugendliche mit Hörbehinderungen. Sie stellt die einzige Studie zur Prävention von sexualisierter Gewalt von Kindern und Jugendlichen mit Körperbehinderungen dar, die mithilfe der beschriebenen Suchstrategien aufgefunden werden konnte. Nach Angaben der Autor:innen ist ihr Präventionstraining „STARK mit SAM“ das erste seiner Art. Das Training fußt auf dem Projekt „Vorbeugen und Handeln - Sexueller Missbrauch an Kindern und Jugendlichen mit Behinderung “ (SeMB). Dieses Projekt wandte sich mit einer prospektiven Befragungs- und Interventionsstudie zu sexualisierter Gewalt an Kinder und Jugendliche mit körperlicher Behinderung, Intelligenzminderung und Hörbehinderung. Es wurden insgesamt drei Trainings mit weitgehend identischen Inhalten konzipiert, die jeweils an die spezifischen Voraussetzungen von Kindern mit körperlicher Behinderung, Intelligenzminderung und Hörbehinderung angepasst sind. Das Präventionstraining liegt als Gruppentraining für Kinder (8-12 Jahre) und Jugendliche (13-17 Jahre) vor.

In der Evaluationsstudie (Urbann et al. 2020) durchliefen 92 Kinder mit Hörbehinderungen im Alter von 8-12 Jahren das Präventionsprogramm „STARK mit SAM“. Sie wurden unterteilt in eine Experimental- und eine Wartegruppe und ihr Präventionswissen wurde vor, direkt nach und sechs Monate nach der Intervention getestet. Dabei zeigte sich ein positiver Effekt des Präventionsprogramms.

Das Manual von „STARK mit SAM“ ist aktuell angekündigt als beziehbar ab Juni $2021^{2}$. Auszüge sind aktuell online und über eine Dissertationsschrift verfügbar (Bienstein und Verlinden 2018; Urbann 2018).

Des Weiteren gibt es Programme und Materialien (vgl. Chodan et al. 2014a, 2014b), deren Wirksamkeit noch nicht untersucht bzw. deren Evaluation nicht in wissenschaftlichen Fachzeitschriften publiziert wurde. Den Einsatz solcher Präventions- oder Interventionsprogramme, deren Evidenz noch nicht gezeigt wurde, bezeichnen Yeater und O‘Donohue (1999) als deliver-and-hope-Strategie, die möglicherweise Ressourcen verschwendet und im schlimmsten Fall sogar schaden kann.

Und auch bei den vorgestellten Programmen, deren Wirksamkeit zwar jeweils durchaus untersucht wurde, ist aufgrund der Methoden der Studien die Validität der Ergebnisse nicht abschließend geklärt, da nur eine Studie eine ausreichend große Stichprobengröße mit verhaltensnahen Erhebungsmaßen verknüpfte - und deren Ergebnisse unterstreichen, dass Programme, die sich an Kinder und Jugendliche mit Behinderungen wenden (hier gezeigt für Kinder und Jugendliche mit Intelligenzminderung; Reis et al., eingereicht zur Publikation), alleinig keinen ausreichenden Schutz bieten. Es bleibt daher der eingangs zitierte Leitsatz bestehen, dass Präven-

2 https://www.hogrefe.com/de/shop/praevention-sexuellen-miss brauchs-an-kindern-und-jugendlichen-mit-behinderung-75876.html tion sexualisierter Gewalt mehrdimensional ist und verschiedene, ineinandergreifende Ansätze umfasst (Brown et al. 1994), zu denen auch die präventive Arbeit mit potenziellen Tatpersonen gehört sowie institutionelle und gesellschaftliche Ansätze, zu denen leider bislang keine Wirksamkeitsstudien zu konkreten Ansätzen aufgefunden werden konnten.

\section{Therapeutische Optionen für Kinder und Jugendliche mit Behinderung, die sexualisierte Gewalt erfahren haben}

Kommt es zu sexualisierter Gewalt, sollte diese beendet werden und es sollten Hilfen für die betroffenen Kinder oder Jugendlichen angeboten und ggf. implementiert werden. Es sollte überlegt statt übereilt gehandelt werden, wobei eine räumliche Trennung von Betroffenen und Tatperson bei gegenwärtiger sexualisierter Gewalt möglichst zeitnah erfolgen sollte. Auch hier steht- wie bei allen Kindern und Jugendlichen - das Kindeswohl im Vordergrund.

Die folgende Analyse der Versorgungssituation für die Behandlung von Kindern und Jugendlichen mit Behinderungen, die sexualisierte Gewalt erlebt haben, erfolgt in drei Abschnitten getrennt nach der Art der Behinderung, da Interventionen auf diese zugeschnitten sein müssen. Zunächst werden Studien berichtet, die nicht zwischen den Behinderungsformen differenzieren bzw. verschiedene Behinderungsformen betrachten. Danach wird die Situation beschrieben, die für Kinder und Jugendliche mit einer Intelligenzminderung besteht; abschließend folgt die Analyse der Versorgungssituation für Kinder und Jugendliche mit Körperbehinderungen.

Mikton et al. (2014) unternahmen eine umfangreiche Literaturrecherche, die nur zehn Publikationen ausfindig machte, die Programme für Personen mit Behinderungen - darunter acht Studien zu Lernbehinderungen und Intelligenzminderung - evaluieren. Dabei unterschieden die Autoren nicht zwischen Prävention und Intervention, sodass mindestens vier der zehn Studien Präventionsprogramme statt Interventionsprogramme evaluierten. Nur zwei Studien betrachteten sexualisierte Gewalt gegen Kinder und Jugendliche: Ahn (2004) schloss 15 Eltern von Vorschulkindern mit Behinderungen ein und Cowen und Reed (2002) schlossen 148 Familien und 265 Kinder mit Behinderungen ein. Die Qualität der Methodiken aller Studien war schwach (gemessen mit dem Quality Assessment Tool QATQS; EPHPP 2013) und eine Wirksamkeit konnte nach der Berücksichtigung von Vorurteilsrisiken (Bias) für keines der Programme festgestellt werden.

Auch seit 2014 konnte diese Lücke nicht geschlossen werden. Unter den vier aufgefundenen Publikationen ist neben dem Review von Mikton et al. (2014) ein weiteres narratives Review (Hernon et al. 2014), das auf Rechte und Barrieren fokussiert und kaum Originalstudien benennt. Die zwei Studien, die im Kapitel zu den Interventionen genannt werden, berichten jeweils Erkenntnisse aus Interviews mit 61 Mitarbeiter:innen von Anlaufstellen für Kinder und Jugendliche mit Behinderungen und Kinderschutz (Taylor et al. 2014, zit. n. Hernon et al. 2014) bzw. Erkenntnisse aus einer „thematischen Inspektion“ von zwölf Anlaufstellen (Ofsted Report von 2012, zit. n. Hernon et al. 2014). Auch Franklin und Smeaton (2017) berichten von Interviews zur Versorgungssituation von Menschen mit verschiedenen Behinderungen, ohne die konkreten Versorgungssituationen genauer zu untersuchen. 
Weiterhin sollte neben direkten Hilfsangeboten an die Betroffenen folgendes Interventionsrepertoire bedacht werden:

- Gespräche im Team führen,

- externe Stellen hinzuziehen,

- rechtliche Schritte einleiten,

- eine medizinische Untersuchung durchführen lassen,

- die Aufsicht verstärken und die Tatpersonen entlassen.

(Noack und Schmidt 1994).

Allzu häufig werden übergriffige Mitarbeiter:innen jedoch nur versetzt, um kein Aufsehen zu erregen und das vermeintliche Image der Institution zu wahren. Offenbar wird oft davon ausgegangen, dass eine Institution in den Augen der Gesellschaft „gefährlicher“ und unsicherer wirkt, wenn ein Fall von sexualisierter Gewalt publik gemacht wird - wobei das Gegenteil der Fall sein sollte, denn ein offensiver und konsequenter Umgang der Leitungsebene mit diesem Thema erscheint den Autor:innen als ein protektiver Faktor. Hier bedarf es dringend der Erforschung dieser Phänomene sowie weiterer Aufklärung der Bevölkerung und einer transparenten Kultur des Berichtens.

Es konnten keine Studien aufgefunden werden, die die Wirksamkeit von Programmen für die Behandlung von Kindern und Jugendlichen mit einer Intelligenzminderung zur Verarbeitung von sexualisierter Gewalt evaluiert haben. Wirksame, auf die Bedürfnisse der Zielgruppen zugeschnittene, vorzugsweise manualisierte und standardisierte Therapieprogramme fehlen somit weiterhin. Stattdessen werden geläufige Therapien, wie Traumatherapie oder die Arbeit mit Körperreaktionen, methodisch und sprachlich vereinfacht durchgeführt. Aber auch hier fehlen systematische Studien zur Wirksamkeitsüberprüfung: Ein aktuelles Review von Chen et al. (2018) bestätigt zwar die Wirksamkeit von EMDR bei Kindern; deren Moderation durch das Vorliegen von Behinderungen, darunter insbesondere einer Sehbehinderung oder Intelligenzminderung, wurde nicht untersucht. Und während Keesler (2014) zwar eine Integration von Trauma-Informed Care (einem systemorientierten Modell für die Bereitstellung von Versorgungsangeboten für Menschen mit traumatischen Lebenserfahrungen) und den besonderen Bedarfen von Kindern und Jugendlichen mit Lernbehinderung oder Intelligenzminderung ankündigt, bleibt diese doch aus. Der beschriebene Mangel an speziellen Materialien beziehungsweise Programmen ist vermutlich ein Grund dafür, warum leider nur sehr wenige Beratungsstellen therapeutische Angebote für Kinder und Jugendliche mit Intelligenzminderung zur Verarbeitung von sexueller Gewalt machen. Das meistens erforderliche Hinzuziehen externer Stellen, um den Betroffenen Gespräche und anderweitige Therapien zugänglich zu machen, gestaltet sich in der Umsetzung daher leider oft schwierig. Es ist somit nach unserer Recherche aktuell kein einziges standardisiertes und evaluiertes Vorgehen verfügbar, das auf diese Zielgruppe zugeschnitten ist. Dieses Problem zeigt sich auch im neuen Leitlinien-Manuskript für die Leitlinie zur Intelligenzminderung, welches hochaktuell - am 14.04.2021 - bei der Arbeitsgemeinschaft der Wissenschaftlichen Medizinischen Fachgesellschaften e. V. (AWMF) zur Begutachtung eingereicht worden ist. Darin können aufgrund des Mangels an Forschung in diesem Bereich ebenfalls keine therapeutischen Optionen für den Umgang mit sexualisierter Gewalt gelistet oder beschrieben werden (Häßler et al., eingereicht zur Publikation).
Etwas optimistischer ist die Situation für Kinder und Jugendliche mit körperlicher Behinderung. Die Webseite von gegen-missbrauch e. V. bietet eine Umkreissuche nach barrierearmen Anlaufstellen (z. B. mit Gebärdensprache, für Sehbehinderungen oder rollstuhlgerecht; https://www.gegen-missbrauch.de/hilfeadressen/). Viele Beratungsstellen bieten zudem eine Online-Beratung an, z. B. die Beratungsstelle des Mädchenhauses Bremen e. V., was Kindern und Jugendlichen mit körperlichen Behinderungen entgegenkommt, die in ihrer Mobilität eingeschränkt oder hörgeschädigt sind.

Auch hier mangelt es jedoch an Studien zur Evaluation dieser Interventionen. Lediglich Jones et al. (2017) berichtet eine qualitative Studie, die das Führen geleiteter (strukturierter) Interviews direkt mit Kindern und Erwachsenen mit einer Hörbehinderung untersucht. Die Studie identifiziert als Ergebnis drei Möglichkeiten, gehörlose Kinder zu unterstützen: die Fähigkeit von Erwachsenen, sexualisierte Gewalt zu erkennen, eine unterstützende Reaktion auf Offenlegungen und den Zugang zu registrierten Dolmetscher:innen.

Ihre Empfehlungen umfassen:

- Aufklärung und Sensibilisierung von Praktizierenden, Kindern, Eltern und Betreuer:innen;

- Bekämpfung der Isolation von gehörlosen Kindern und Kindern mit Behinderungen;

- Bereitstellung umfassender Unterstützungsdienste, die ganzheitlich auf die Bedürfnisse der Kinder eingehen;

- Sicherstellen, dass die Stimme der Kinder gehört wird;

- routinemäßiger Zugang zu registrierten Dolmetscher:innen für gehörlose Kinder im Rahmen von allgemeinen und spezialisierten Diensten;

- Maßnahmen zur Bekämpfung von Behinderungen auf lokaler und institutioneller Ebene.

\section{Besonderheiten bei der rechtlichen Verfolgung sexueller Übergriffe}

Anhaltspunkte für die Aufdeckung können verbale oder nonverbale Äußerungen der Betroffenen, Verhaltensänderungen infolge der sexualisierten Gewalt oder Tatzeug:innen sein. Verbale Äußerungen können durch mit der Behinderung verbundene Sprachbarrieren erschwert sein. Das verbreitete Vorurteil der Unglaubwürdigkeit von behinderten Kindern verhindert darüber hinaus, dass deren verbale sowie nonverbale Äußerungen ernst genommen werden, womit notwendige Interventionen oft ausbleiben (Bay 2012; Gerdtz 2003). Verhaltensänderungen infolge sexualisierter Gewalt, z. B. aggressives Verhalten, werden häufig als Ausdruck der Behinderung fehlgedeutet und somit nicht erkannt (Cooke 2000). Da das Leben in Einrichtungen und die mit Behinderungen oft verbundene soziale Isolation die Wahrscheinlichkeit vermindern, dass außenstehende Personen Zeug:innen der Straftat werden (Bay 2012; Seligmann 1996), sind auch Tatzeug:innen oft kein ausreichender Anhaltspunkt für die Aufdeckung von sexuellen Straftaten gegen Menschen mit Behinderung.

Selbst in den aufgedeckten Fällen gibt es nur wenige gerichtliche Verhandlungen. Dies ist zum einen in der erschwerten Zugänglichkeit polizeilicher Dienststellen (z. B. nicht rollstuhlgerechte Gebäude) und in kommunikativen Barrieren begründet (Bay 2012; Degener 1994). Zum anderen kann insbesondere bei Kindern und Jugendlichen mit Intelligenzminderung die Erinnerungsfähigkeit 
beeinträchtigt sein; bei Kindern und Jugendlichen mit Taubheit oder Hörbehinderungen hingegen mangelt es oft am Zugang zu zugelassenen Interpret:innen (Jones et al. 2017).

Wie auch für Kinder ohne Behinderungen gilt, dass die Anforderungen eines Prozesses und die hohe Beweislast eine große Belastung darstellen, insbesondere, wenn sie weder traumasensibel noch betroffenengerecht durchgeführt werden. Die oft überfordernde Situation während und nach einem Strafprozess erfordert daher zwingend die Einhaltung der Rechte von Kindern und Jugendlichen im Rechtssystem (Marquardt 2014) und psychosoziale Prozessbegleitung (vgl. Seligmann 1996).

\section{Zusammenfassung und Diskussion}

Im Jahr 2001 bezeichneten Fegert und Müller die wissenschaftliche und praxisnahe Literatur als „sehr schwer zugänglich“ und die öffentliche Diskussion als „noch in den Kinderschuhen“ stehend (Fegert und Müller 2001: 7 f.). Im Jahr 2014 erschien mit dem umfassenden Buch „Sexueller Missbrauch von Kindern und Jugendlichen" ein Werk, das mit seinen etwa 500 Seiten Lehrbuchcharakter hat (Fegert et al. 2014). In diesem wird der „Sexuelle Missbrauch von Kindern und Jugendlichen mit Behinderung“ mit einem Forschungsüberblick dargestellt (Chodan et al. 2014b), welcher die bestehenden Lücken insbesondere in der Versorgung herausstellt. Der vorliegende Beitrag aktualisiert diesen früheren Forschungsüberblick und kann dabei die kritische Beurteilung von Fegert und Müller (2001) noch immer nicht entkräften.

Lediglich 39 deutsch- und englischsprachige Publikationen konnten identifiziert werden, die seit 2014 zum Thema der sexualisierten Gewalt von Kindern und Jugendlichen mit Behinderung publiziert wurden. Viele dieser Studien zeichnen sich durch geringe methodische Qualität aus (z. B. kleine Stichproben, nicht validierte Instrumente, fehlende Kontrollgruppen).

Von den 39 Studien beschäftigt sich nur ein Drittel mit der Versorgung. Von den restlichen 26 Studien greifen viele Forschungsfragen auf, die längst beantwortet wurden, wie die in dieses Review bewusst eingefügten älteren Quellenverweise belegen. Zwar ist es von Zeit zu Zeit notwendig, Erhebungen zu Prävalenzen zu wiederholen, um die Gültigkeit in einer sich wandelnden Kultur zu überprüfen, aber die Fülle an Studien - insbesondere im Vergleich zum Mangel an Studien zur Versorgung - lässt sich damit nicht rechtfertigen. Stattdessen sollten die Ressourcen in Studien zur verbesserten Versorgung fließen, um hier wichtige Erkenntnislücken zu schließen, z. B.: Wie effektiv sind Präventionsprogramme, die sich direkt an die Kinder und Jugendlichen mit Behinderungen wenden, wenn die Effektivität an einer ausreichend großen Stichprobe mit verhaltensnahen, realitätsnahen Mitteln erhoben wird? Können die erlernten Strategien tatsächlich in realen Situationen von sexualisierter Gewalt angewendet werden (ökologische Validität)? Welche Maßnahmen sorgen auf institutioneller Ebene für eine Reduktion des Risikos für sexualisierte Gewalt? Wie kann das Umfeld für sexualisierte Gewalt an Kindern und Jugendlichen mit Behinderungen sensibilisiert werden, und wie kann es dazu befähigt werden, diese zu erkennen und zu beenden? Wie können Kinder und Jugendliche, die sexualisierte Gewalt erlebt haben, effektiv gestützt werden, sowohl rechtlich wie therapeutisch wie sozial?
Wie können emotionale und Verhaltensprobleme, die daraus erwachsen können, effektiv behandelt werden?

\section{Erkenntnisse zur Epidemiologie}

Das Risiko für Kinder und Jugendliche mit Behinderung, von sexueller Gewalt betroffen zu werden, ist größer als im Bevölkerungsdurchschnitt (Mailhot Amborski et al. 2021). Die größte Einzelstudie ist nach wie vor die von Sullivan und Knutson (2000) an über 40000 Kindern und Jugendlichen (davon 3262 Kinder und Jugendliche mit Behinderungen). Wie die Studie von Sullivan und Knutson (2000) eindrücklich zeigt, stellt die Form der Behinderung einen wichtigen Risikofaktor dar, wobei hier widersprüchliche Ergebnisse in der Literatur zu finden sind (so sind bei Mailhot Amborski et al. (2021) und der Studie des BMFSFJ (2013) Kinder und Jugendliche mit sensorischen Behinderungen am häufigsten betroffen, während bei Sullivan und Knutson (2000) Kinder und Jugendliche mit Verhaltensstörungen am häufigsten betroffen sind). Zahlreiche Risikofaktoren, die sich bisher gesellschaftlich als (nicht zwangsläufige) Reaktionen ergeben, wie soziale Isolation, Fremddominierung, eingeschränkte Kommunikationsfähigkeit, geringe Akzeptanz des eigenen Körpers und mangelhafte Sexualaufklärung, kumulieren im Lebensumfeld von Kindern und Jugendlichen mit Behinderungen. Das erhöhte Risiko setzt sich demnach aus zahlreichen Risikofaktoren zusammen, die sich aus den besonderen Lebensstrukturen und dem gesellschaftlichen Umgang mit Behinderungen ergeben.

Unter den Artikeln zur Epidemiologie ist keine Einzelstudie, die die umfassende Studie von vor 20 Jahren (Sullivan und Knutson 2000) ausreichend ersetzen könnte, da weder die Stichprobengröße noch der Grad der Differenzierung (von Behinderungs- sowie Misshandlungsformen) noch einmal erreicht wird. Es lässt sich nach wie vor festhalten, dass Kinder und Jugendliche mit einer Behinderung signifikant häufiger von (sexuellen) Misshandlungen betroffen sind als Kinder und Jugendliche ohne Behinderung - eine alte Erkenntnis, die nun aber auch durch eine Metaanalyse (Mailhot Amborski et al. 2021) gestützt wird.

Zudem handelt es sich in der Mehrzahl der Fälle um mehrmalige Übergriffe, die über längere Zeit andauern, durch vertraute (zumeist männliche) Tatpersonen. Eine chronische bzw. wiederholte Erfahrung sexualisierter Gewalt durch eine bekannte und vertraute Person wirkt pathogener als ein einmaliger Vorfall sexualisierter Gewalt durch eine unbekannte Tatperson (Mansell und Sobsey 2001), weshalb dringend Bedarf an Präventions- und Interventionsangeboten besteht.

\section{Erkenntnisse zur Versorgung}

Die wichtigsten Befunde aus den Kapiteln zur Prävention von sexualisierter Gewalt gegen Kinder und Jugendliche mit Behinderungen und zur Intervention nach dem Erleben sexualisierter Gewalt lassen sich wie folgt zusammenfassen: Die Prävention sollte - wie auch für Kinder und Jugendliche ohne Behinderungen - mehrdimensional erfolgen und neben Trainings, die die Kinder und Jugendlichen stärken sollen, auch Tatpersonenprävention sowie Prävention auf institutioneller und gesellschaftlicher Ebene umfassen. Innerhalb der Präventionsprogramme, die sich direkt an Kinder und Jugendliche mit Behinderungen wenden, sprechen die Ergebnisse der Studien dafür, dass diese Form der Prävention effektiv ist und in die umfassendere, multidimensionale Prävention 
mit eingebunden werden kann. Unter den sieben neueren Arbeiten zur Prävention sind vier Evaluationsstudien, von denen drei durch gravierende methodische Mängel (z. B. Fehlen einer Kontrollgruppe, unzureichende Stichprobengröße, Fehlen valider Erhebungsinstrumente) gekennzeichnet sind. Zumindest wurden dank der Förderung des BMBF zwei Programme für die Prävention von sexualisierter Gewalt für Kinder und Jugendliche mit körperlichen Behinderungen und/oder Intelligenzminderung (insbesondere Gehörlosigkeit und leichte Intelligenzminderung) entwickelt und evaluiert, die methodisch einer deutlich höheren Evidenzklasse entsprechen. Hier erwiesen sich behaviorale und spielerische Elemente (z. B. Rollenspiele) und realitätsnahe Szenarien (um den Transfer zu erleichtern) als besonders wirksame Präventionsmethoden. Insbesondere die aufwändige Evaluation eines der Präventionsprogramme mit realitätsnahen und verhaltensnahen $\mathrm{Er}$ hebungsmethoden zeigt jedoch auch die Grenzen der Wirksamkeit desselbigen (Reis et al., eingereicht zur Publikation), sodass nicht auszuschließen ist, dass in tatsächlichen Gewaltsituationen auf die gelernten Kompetenzen nicht ausreichend zurückgegriffen wird. Dieses Ergebnis untermauert die bereits mehrfach erhobene Forderung, Prävention multidimensional zu gestalten, indem verschiedene Präventionsansätze ineinandergreifen (vgl. Brown et al. 1994). An dieser Stelle wird allerdings der Mangel an Wirksamkeitsstudien zu anderen Präventionsansätzen deutlich: Obwohl sich die Autorinnen Conen und Enders bereits 1995 intensiv mit der Frage befasst haben, welche institutionellen Rahmenbedingungen das Risiko für sexualisierte Gewalt erhöhen (zit. n. Bange 2014), lagen bis 2010 keine empirischen Belege dafür vor (Bundschuh 2010, zit. n. Bange 2014). Dies ist eine Situation, die sich nach der aktuellen Literaturrecherche scheinbar nicht geändert hat. Conen und Enders (1995, zit. n. Bange 2014) identifizieren überstrukturierte Einrichtungen - mit rigiden Strukturen und autoritärem Leitungsstil - und wenig strukturierte Einrichtungen - mit einem Mangel an klaren Regeln und Entscheidungen und einer schwachen Leitung - als besonders riskant. Bange (2014) fügt diesen noch ein weiteres Risikoprofil hinzu, nämlich das der weitgehend geschlossenen Einrichtungen - mit einer großen Nähe zwischen den zu Betreuenden und den Fachkräften, einer scharfen Abgrenzung gegenüber anderen sozialen Systemen und oft einer charismatischen Leitung. Es ist dringend an der Zeit, die Risiko- und Schutzfaktoren, die sich daraus ableiten lassen (Bange 2014), einer Evidenzprüfung zu unterziehen. Zwar ist das endgültige Ziel ein Ineinandergreifen verschiedener Präventionsansätze, aber zum aktuellen Zeitpunkt sind für ein sauberes wissenschaftliches Arbeiten (zum Ausschluss von Störeinflüssen und alternativen Erklärungsmodellen) Einzelstudien in diesen Bereichen (institutionelle Prävention, aber auch Prävention auf gesellschaftlicher Ebene und Tatpersonenprävention) vonnöten.

Bezogen auf Interventionen ließen sich in der aktuellen Recherche nur vier Publikationen auffinden, von denen eine den Status quo beschreibt und zwei Überblicksarbeiten darstellen (eine eher beschreibend, ohne konkrete Studien zu benennen, und die andere mit dem Ergebnis, dass die zwei auffindbaren Studien gravierende methodische Mängel aufweisen). Somit bleibt eine einzige Studie, die sich konkret mit der Intervention nach sexualisierter Gewalt bei Kindern und Jugendlichen mit Behinderungen (hier: Hörbehinderung) befasst, wobei auch diese in ihren Empfehlungen sehr allge- meingültig bleibt und z. B. die „Bereitstellung umfassender Unterstützungsdienste, die ganzheitlich auf die Bedürfnisse der Kinder eingehen“ (Jones et al. 2017: 762, übersetzt von WC), als Interventionsansatz empfiehlt.

Wirksame, auf die Bedürfnisse der Zielgruppen zugeschnittene, vorzugsweise manualisierte und standardisierte Therapieprogramme fehlen somit weiterhin für alle Formen von Behinderungen.

Wie die Studie von Sullivan und Knutson (2000) eindrücklich zeigt, stellt die Form der Behinderung einen wichtigen Risikofaktor dar. Der Unterschiedlichkeit von Behinderungen wird jedoch insgesamt zu wenig Rechnung getragen; so differenzieren viele Studien nicht zwischen den Behinderungsformen oder es werden Angebote herangezogen, die für Kinder und Jugendliche ohne Behinderungen konzipiert sind, was in diesem Artikel sowohl für kognitive Behinderungen (EMDR für Kinder und Jugendliche mit Intelligenzminderung) als auch für körperliche/sensorische Behinderungen gezeigt wurde. Für letztere werden oft Anlaufstellen lediglich barrierearm ausgestattet. Dies ist aber nicht damit gleichzusetzen, dass sie auf die besondere Lebenssituation von Kindern und Jugendlichen zugeschnitten sind. Um es provokant zu formulieren: Eine Rampe oder ein:e Dolmetscher:in für Gebärdensprache reichen nicht aus, um die komplexe Lebenswelt dieser Kinder und Jugendlichen, ihr Recht auf selbstbestimmte Sexualität, ihr Recht auf Teilhabe, ihre soziale Isolation und Stigmatisierung oder die Abhängigkeit von anderen abzubilden. Stattdessen gilt es, diese Aspekte gezielt in beratenden und therapeutischen Angeboten zu berücksichtigen. Es bedarf nicht nur dringend weiterer gezielter Forschung, sondern weiterhin auch ruhiger Aufmerksamkeit und eines kulturellen Wandels, um die Bedarfe dieser großen Gruppe - immerhin 10\% aller Kinder und Jugendlichen (Maclean et al. 2017) - ausreichend zu berücksichtigen.

\section{Limitationen}

Für dieses Review wurden nur deutsch- und englischsprachige Publikationen berücksichtigt. Da die Publikationslandschaft nach wie vor sehr karg ist, war es nur für den Bereich der Prävalenzen möglich, eine Metaanalyse einzuschließen. Alle anderen Kapitel tragen Studienergebnisse möglichst strukturiert zusammen, haben jedoch nicht den Anspruch, diese statistisch zusammenzufassen und zu bewerten.

\section{Ausblick}

Auf Basis des vorliegenden Reviews sollten vordringlich Forschungslücken im Bereich der Versorgung geschlossen werden. Die Erkenntnisse zur Prävalenz und damit zum erhöhten Risiko bei Kindern und Jugendlichen, von sexualisierter Gewalt betroffen zu werden, konnten über Jahre und Länder hinweg in verschiedenen Studien untermauert werden, wie jüngst in einer Metaanalyse zusammengefasst (Mailhot Amborski et al. 2021). Präventionsprogramme und Interventionen, die dieses Risiko senken bzw. negative Folgen abmildern sollen, sollten gemäß diesem Erkenntnisstand, der mindestens seit den 1990er-Jahren besteht, bereits längst entwickelt und erforscht worden sein. Doch international fanden sich nur neun Evaluationen von Präventionsprogrammen (davon sechs seit 2017, wobei eine dieser Publikationen ein Studienprotokoll ist und die Ergebnisse dieser Studie noch nicht veröffentlicht wurden) - von denen alle Trainingsprogramme untersuchen, die sich 
direkt an Kinder und Jugendliche mit Behinderungen wenden, sodass Evaluationen institutioneller Ansätze beispielsweise gänzlich fehlen, obwohl hier ein erheblicher Teil sexualisierter Gewalt zu verorten ist - und nur eine Evaluation einer Intervention. Nur diese Intervention und eines der Präventionsprogramme wenden sich an Kinder und Jugendliche mit Hörbehinderungen; die übrigen Präventionsprogramme richten sich an Kinder und Jugendliche mit Intelligenzminderung. Für zahlreiche Behinderungsformen gibt es daher noch keinerlei zugeschnittene und evaluierte, möglichst standardisierte und manualisierte Präventionsprogramme oder Interventionen.

Erschwerend kommt hinzu, dass fast alle Evaluationsstudien gravierende methodische Mängel aufweisen, darunter zu geringe Stichprobengrößen, Fehlen von echten Kontrollgruppen, fehlende Erhebung der Unbedenklichkeit und geringe ökologische Validität der Erhebungsmethoden. Die Methodologie sollte daher eine Bestimmung der Mindeststichprobe enthalten, um eine ausreichende Power und eine Generalisierung der Ergebnisse von der Stichprobe auf die Population zu ermöglichen. Die Einführung einer Kontrollbedingung sichert zudem, dass mögliche positive Effekte nicht fälschlicherweise der Intervention zugeschrieben werden. Lerneffekte, z. B. durch wiederholte emotional intensive Messungen, sollten von den trainingsinhärenten Lerneffekten ausgeschlossen werden. Mehr noch erfordert gerade die hohe Wahrscheinlichkeit von reinen Beziehungseffekten die Einführung einer aktiven Kontrollbedingung, die in Art und Dosis der Beziehungsarbeit vergleichbar ist. Ein weiterer Aspekt, den es zu beachten gilt, ist die Überprüfung der Unbedenklichkeit der Präventions- und Interventionsprogramme. Dieser ethisch wichtige Aspekt wird häufig nicht beachtet; ein Beispiel für dessen Beachtung ist aber das Projekt SeMB, in dem Ängste miterhoben sind. Es muss immer davon ausgegangen werden, dass Teilnehmende an Präventionsprogrammen gegebenenfalls bereits sexualisierte Gewalt erlebt haben. In diesem Fall könnte ein solches Angebot retraumatisierend oder aufdeckend sein, worauf Trainer:innen vorbereitet sein müssen, um angemessen reagieren zu können. Zukünftige Forschungen sollten hier besondere Sorgfalt verwenden, da in sensiblen Forschungsbereichen wie diesem sehr unterschiedliche intercurrent events auftreten können, die die Evaluationsergebnisse nachhaltig beeinflussen. Damit sind $\mathrm{Er}$ eignisse gemeint, die nach der Randomisierung auftreten und die Adhärenz beeinflussen, wie etwa Trainingsabbrüche aufgrund von Schamgefühlen. Die Validität der Erhebung kann durch eine größere Realitätstreue der Erhebungsinstrumente erreicht werden. Insbesondere bei den Studien, die Präventionsprogramme evaluieren, fällt immer wieder auf, dass präventives Verhalten erlernt werden soll, aber nur Präventionswissen erhoben wird. Dies ist beispielsweise beim oft eingesetzten What-If Situations-Test der Fall, der lediglich verbale Angaben der Proband:innen dazu erhebt, welches Verhalten sie an den Tag legen würden, ohne dass überprüft wird, ob sie dieses Verhalten in einer konkreten Situation wirklich umsetzen würden oder könnten. Daher gilt insbesondere bei der Evaluation von Präventionsprogrammen: Wenn im Programm Verhalten erlernt werden soll, sollte in der Evaluation auch Verhalten gemessen werden.

Die Validität der Ergebnisse zu erhöhen, ist nicht nur eine Frage der Methodologie, sondern auch der Ethik. Ein falsch positives Ergebnis, also das Ergebnis, dass ein Präventions- oder Interventi- onsprogramm positive Effekte erzielt, obwohl dies nicht der Fall ist (z. B. weil die Proband:innen das vorab angekündigte Verhalten eben doch nicht in der konkreten Situation umsetzen), könnte den Teilnehmer:innen schaden, denn das Umfeld kann nun fälschlicherweise vom Schutz der Teilnehmer:innen ausgehen. Eventuell wird dann von den Teilnehmer:innen erwartet, dass diese nun aktiv Vorfälle berichten (wie es viele Präventionsprogramme aus gutem Grund lehren), womit externe Berichtsroutinen abgewertet werden könnten.

Deshalb sollten neben Präventions- und Interventionsprogrammen, die sich direkt an Kinder und Jugendliche wenden, auch institutionelle und andere Präventionsroutinen weiterentwickelt und erforscht werden.

\section{Interessenkonflikt}

Die Autor:innen geben an, dass kein Interessenkonflikt besteht.

Literatur $^{3}$

Ahn H. The Effect of a Child Abuse Prevention Program for Parents with Disabled Children. JKANA 2004; 34: 663-672

*Araten-Bergman T, Bigby C, Ritchie G. Literature Review of Best Practice Supports in Disability Services for the Prevention of Abuse of People with Disability. Report for the Disability Services Commissioner. Melbourne: Living with Disability Research Centre, La Trobe University 2017

*Austin A, Herrick H, Proescholdbell S, Simmons J. Disability and Exposure to High Levels of Adverse Childhood Experiences: Effect on Health and Risk Behavior. N C Med J 2016; 77: 30-36

Baladerian NJ. Sexual Abuse of People with Developmental Disabilities. Sex Disabil 1991; 9: 323-335

Balogh R, Bretherton K, Whibley S, Berney T, Graham S, Richold P, Worsley C, Firth H. Sexual Abuse in Children and Adolescents with Intellectual Disability. J Intellect Disabil Res 2001; 45: 194-201

Bange D. Gefährdungslagen und Schutzfaktoren im familiären und institutionellen Umfeld in Bezug auf sexuellen Kindesmissbrauch. In: Fegert JM, Hoffmann U, König E, Niehues J, Liebhardt H, Hrsg. Sexueller Missbrauch von Kindern und Jugendlichen: Ein Handbuch zur Prävention und Intervention für Fachkräfte im medizinischen, psychotherapeutischen und pädagogischen Bereich. Berlin, Heidelberg: Springer 2014; 137-144

Bange D, Enders U. Wir sind nicht die einzigen*. Fakten zum sexuellen Missbrauch in Institutionen. In: Enders U, Hrsg. Grenzen achten: Schutz vor sexuellem Missbrauch in Institutionen. Ein Handbuch für die Praxis. Köln: Kiepenheuer \&Witsch 2012; 15-29

Bay Y. Sexualisierte Gewalt gegenüber Mädchen und Frauen mit Behinderung. Selbstbehauptung und Selbstverteidigung als präventive Maßnahme. Unveröffentlichte Staatsprüfung, Pädagogische Hochschule Ludwigsburg in Verbindung mit der Universität Tübingen mit Sitz in Reutlingen 2012

Becker M. Sexuelle Gewalt gegen Mädchen mit geistiger Behinderung: Daten und Hintergründe. Heidelberg: edition schindele 1995

Belsky J. Child Maltreatment: An Ecological Integration. Am Psychol 1980; 35: 320

3 Die im Review eingeschlossenen 39 Publikationen sind im Literaturverzeichnis mit einem Sternchen $\left({ }^{*}\right)$ markiert. 
Bienstein P, Verlinden K, Hrsg. Prävention von sexuellem Missbrauch an Menschen mit geistiger Behinderung. Ausgewählte Aspekte. Berlin: Eigenverlag der DGSGB 2018 [Als Online-Dokument: https://www. fk-reha.tu-dortmund.de/IB/cms/Medienpool/Dokumente/DGSGB_ Bienstein_Verlinden.pdf]

[BMFSF]] Bundesministerium für Familie, Senioren, Frauen und Jugend. Lebenssituation und Belastungen von Frauen mit Beeinträchtigungen und Behinderungen in Deutschland. Kurzfassung. Broschüre. Berlin: BMFSFJ 2013

Brill W. Sexuelle Gewalt gegen behinderte Menschen - ein Überblick über den aktuellen Stand der Diskussion. Behindertenpädagogik 1998; 37 155-172

*Brisma S, Amir N. Preventive Treatment on Sexual Abuse Survivor with Mental Retardation. AMCR 2020; 1: 15-19

Bronfenbrenner U. Toward an Experimental Ecology of Human Development. Am Psychol 1977; 32: 513-531

Brown H, Hunt N, Stein J. Alarming But Very Necessary: Working with Staff Groups around the Sexual Abuse of Adults with Learning Disabilities. J Intellect Disabil Res 1994; 38: 393-412

Bruder C, Stenfert Kroese B. The Efficacy of Interventions Designed to Prevent and Protect People with Intellectual Disabilities from Sexual Abuse: A Review of Literature. J Adult Prot 2005; 7: 13-27

*Büyükbayraktar ÇG, Er RK, Kesici S. Creating Awareness of Sexual Abuse in Children with Special Education Needs: Depending on the Opinions of Teachers of the Mentally Handicapped. J Educ Train Stud 2018; 6: 151-158

*Byrne G. Prevalence and Psychological Sequelae of Sexual Abuse among Individuals with an Intellectual Disability: A Review of the Recent Literature. J Intellect Disabil 2018; 22: 294-310

${ }^{*}$ Caldas SJ, Bensy ML. The Sexual Maltreatment of Students with Disabilities in American School Settings. J Child Sex Abuse 2014; 23: 345-366

Cederblad M, Dahlin L, Hagnell O, Hansson K. Salutogenic Childhood Factors Reported by Middle-Aged Individuals. Follow-Up of the Children from the Lundby Study Grown Up in Families Experiencing Three or More Childhood Psychiatric Risk Factors. Eur Arch Psychiatry Clin Neurosci 1994; 244: 1-11

*Chen R, Gillespie A, Zhao Y, Xi Y, Ren Y, McLean L. The Efficacy of Eye Movement Desensitization and Reprocessing in Children and Adults Who Have Experienced Complex Childhood Trauma: A Systematic Review of Randomized Controlled Trials. Front Psychol 2018 ; 9: 534

Chodan W, Häßler F, Reis O. Programme zur Prävention von sexuellem Missbrauch von Menschen mit geistiger Behinderung. Prax Kinderpsychol Kinderpsychiatr 2014a; 63: 82-98

*Chodan W, Häßler F, Reis O. A Randomized Controlled Trial on the Effectiveness of a Sexual Abuse Prevention Programme for Girls with Intellectual Disabilities: Study Protocol. Transl Dev Psychiatry 2017; 5: 1407192

Chodan W, Reis O, Häßler F. Sexueller Missbrauch von Kindern und Jugendlichen mit Behinderung. In: Fegert JM, Hoffmann U, König E, Niehues J, Liebhardt H, Hrsg. Sexueller Missbrauch von Kindern und Jugendlichen: Ein Handbuch zur Prävention und Intervention für Fachkräfte im medizinischen, psychotherapeutischen und pädagogischen Bereich. Berlin, Heidelberg: Springer 2014b; 407-419

Cooke P. Final Report on Disabled Children and Abuse. Nottingham: Ann Craft Trust 2000

* Corr C, Santos RM. Abuse and Young Children with Disabilities: A Review of the Literature. J Early Interv 2017; 39: 3-17

Cowen PS, Reed D. Effects of Respite Care for Children with Developmental Disabilities: Evaluation of an Intervention for at Risk Families. Public Health Nurs 2002; 19: 272-283

Degener T. Die sexuelle Gewalt gegen behinderte Frauen. Rechtliche Aspekte. In: Weinwurm-Krause EM, Hrsg. Sexuelle Gewalt und Behinderung. Hamburg: Kovac 1994; 15-24
*Dion J, Paquette G, Tremblay KN, Collin-Vézina D, Chabot M. Child Maltreatment among Children with Intellectual Disability in the Canadian Incidence Study. Am J Intellect Dev Disabil 2018; 123: 176-188

[EPHPP] Effective Public Health Practice Project. Quality Assessment Tool for Quantitative Studies. EPHPP 2013 [Als Online-Dokument: http://www. ephpp.ca/tools.html]

*Euser S, Alink LR, Tharner A, van IJzendoorn MH, Bakermans-Kranenburg MJ. The Prevalence of Child Sexual Abuse in Out-Of-Home Care: Increased Risk for Children with a Mild Intellectual Disability. J Appl Res Intellect Disabil 2016; 29: 83-92

Fegert JM, Hoffmann U, König E, Niehues J, Liebhardt H, Hrsg. Sexueller Missbrauch von Kindern und Jugendlichen: Ein Handbuch zur Prävention und Intervention für Fachkräfte im medizinischen, psychotherapeutischen und pädagogischen Bereich. Berlin, Heidelberg: Springer 2014

Fegert JM, Müller C, Hrsg. Sexuelle Selbstbestimmung und sexuelle Gewalt bei Menschen mit geistiger Behinderung. Bonn: Mebes \& Noack 2001

Fenwick A. Sexual Abuse in Adults with Learning Disabilities. Br J Learn Disabil 1994; 22: 53-56

*Franklin A, Smeaton E. Recognising and Responding to Young People with Learning Disabilities who Experience, or Are at Risk of, Child Sexual Exploitation in the UK. Child Youth Serv Rev 2017; 73: 474-481

Friedrich WN. Behavioral Manifestations of Child Sexual Abuse. Child Abuse Negl 1998; 22: 523-531

*Fry D, Cameron A, Vanderminden J, Lannen P. Child Protection and Disability: Ethical, Methodological and Practical Challenges for Research. Edinburgh: Dunedin Academic Press 2017

Furey EM, Niesen J], Strauch JD. Abuse and Neglect of Adults with Mental Retardation in Different Residential Settings. Behav Interv 1994; 9: 199-211

Garbarino J, Stocking SH. The Social Context of Child Maltreatment. In: Garbarino J, Stocking SH, Hrsg. Protecting Children from Abuse and Neglect: Developing and Maintaining Support Systems for Families. San Francisco: Jossey-Bass 1980; 1-14

Gerdtz M. Auch wir dürfen NEIN sagen! Sexueller Missbrauch von Kindern mit einer geistigen Behinderung. Eine Handreichung zur Prävention. Heidelberg: Universitätsverlag Winter 2003

Hames A. The Effects of Experience and Sexual Abuse Training on the Attitudes of Learning Disability Staff. J Intellect Disabil Res 1996; 40: 544-549

Haseltine B, Miltenberger RG. Teaching Self-Protection Skills to Persons with Mental Retardation. Am J Ment Retard 1990; 95: 188-197

Häßler F, Bienstein P, Buscher M, Caby F, Hennicke K, Hoffmann K, Irblich D, Mendes U, Menzel M, Peters H, Roosen-Runge G. S2k Praxisleitlinie Intelligenzminderung. Berlin: AWMF; eingereicht zur Publikation [Als Online-Dokument: https://www.awmf.org/uploads/ tx_szleitlinien/028-042I_S2k_Intelligenzminderung_2014-12_ver laengert_01.pdf]

Häßler F, Fegert JM, Hrsg. Geistige Behinderung und seelische Gesundheit. Kompendium für Ärzte, Psychologen, Sozialarbeiter und Pflegekräfte. Stuttgart: Schattauer 2005

*Helton J], Gochez-Kerr T, Gruber E. Sexual Abuse of Children with Learning Disabilities. Child Maltreat 2018; 23: 157-165

*Henry N, Flynn A, Powell A. Image-Based Sexual Abuse: Victims and Perpetrators. Trends Issues Crime Crim Justice 2019; 572: 1-19

*Hernon J, Brandon M, Cossar J, Shakespeare T. Recognising and Responding to the Maltreatment of Disabled Children: A Children's Rights Approach. Soc Work Soc Sci Rev 2014; 17: 61-77

Horner-Johnson W, Drum CE. Prevalence of Maltreatment of People with Intellectual Disabilities: A Review of Recently Published Research. Ment Retard Dev Disabil Res Rev 2006; 12: 57-69 
*Jones C, Stalker K, Franklin A, Fry D, Cameron A, Taylor J. Enablers of Help-Seeking for Deaf and Disabled Children Following Abuse and Barriers to Protection: A Qualitative Study. Child Fam Soc Work 2017; 22: $762-771$

*Keesler JM. A Call for the Integration of Trauma-Informed Care among Intellectual and Developmental Disability Organizations. J Policy Pract Intellect Disabil 2014; 11: 34-42

Kendall-Tackett K, Lyon T, Taliaferro G, Little L. Why Child Maltreatment Researchers Should Include Children's Disability Status in Their Maltreatment Studies. Child Abuse Negl 2005; 29: 147-151

* Kim YR. Evaluation of a Sexual Abuse Prevention Program for Children with Intellectual Disabilities. Behav Interv 2016; 31: 195-209

*Kucuk S, Platin N, Erdem E. Increasing Awareness of Protection from Sexual Abuse in Children with Mild Intellectual Disabilities: An Education Study. Appl Nurs Res 2017; 38: 153-158

Lee D, McGee A, Ungar S. The Effectiveness of a Computer-Based Safety Programme for Children with Severe Learning Difficulties. Child Abus Rev 2001; 10: 198-209

Lee YK, Tang CS. Evaluation of a Sexual Abuse Prevention Program for Female Chinese Adolescents with Mild Mental Retardation. Am J Ment Retard 1998; 103: 105-116

Llewellyn MH, McLaughlin TF. An Evaluation of a Self-Protection Skills Program for the Mildly Handicapped. Child Fam Behav Ther 1987; 8: 29-37

Ludlow BL. Contemporary Issues in Sexuality and Mental Retardation. Adv Ment Health Dev Disabil 1991; 4: 1-26

Lumley VA, Miltenberger RG. Sexual Abuse Prevention for Persons with Mental Retardation. Am J Ment Retard 1997; 101: 459-472

Lumley VA, Miltenberger RG, Long ES, Rapp JT, Roberts JA. Evaluation of a Sexual Abuse Prevention Program for Adults with Mental Retardation. J Appl Behav Anal 1998; 31: 91-101

*Maclean MJ, Sims S, Bower C, Leonard H, Stanley FJ, O'Donnell M. Maltreatment Risk among Children with Disabilities. Pediatrics 2017; 139: e20161817

*Mailhot Amborski A, Bussières EL, Vaillancourt-Morel MP, Joyal CC. Sexual Violence against Persons with Disabilities: A Meta-Analysis. Trauma Violence Abuse 2021; doi:10.1177/1524838021995975

Mansell S, Sobsey D. Counselling People with Developmental Disabilities Who Have Been Sexually Abused. Kingston: NADD Press 2001

Mansell S, Sobsey D, Calder P. Sexual Abuse Treatment of Persons with Developmental Disabilities. Prof Psychol Res Pr 1992; 23: 404-409

Marquardt C. Rechte von Kindern und Jugendlichen im Rechtssystem, die Aufgaben von Jugendamt und Familiengericht im Bereich sexueller Missbrauch. In: Fegert JM, Hoffmann U, König E, Niehues J, Liebhardt $\mathrm{H}$, Hrsg. Sexueller Missbrauch von Kindern und Jugendlichen: Ein Handbuch zur Prävention und Intervention für Fachkräfte im medizinischen, psychotherapeutischen und pädagogischen Bereich. Berlin, Heidelberg: Springer 2014; 137-144

Matson JL, Sevin JA. Psychopathology in Persons with Mental Retardation. Oxford, MS: Behavior Intervention Specialists 1988

McCarthy M, Thompson D. A Prevalence Study of Sexual Abuse of Adults with Intellectual Disabilities Referred for Sex Education. J Appl Res Intellect Disabil 1997; 10: 105-124

*McDonnell CG, Boan AD, Bradley CC, Seay KD, Charles JM, Carpenter LA. Child Maltreatment in Autism Spectrum Disorder and Intellectual Disability: Results from a Population-Based Sample. J Child Psychol Psychiatry 2019; 60: 576-584

* Meinck F, Cluver LD, Boyes ME. Household Illness, Poverty and Physical and Emotional Child Abuse Victimisation: Findings from South Africa's First Prospective Cohort Study. BMC Public Health 2015a; 15: 1-13

* Meinck F, Cluver LD, Boyes ME, Mhlongo EL. Risk and Protective Factors for Physical and Sexual Abuse of Children and Adolescents in Africa: A Re- view and Implications for Practice. Trauma Violence Abuse 2015b; 16: 81-107

${ }^{*}$ Mikton C, Maguire H, Shakespeare T. A Systematic Review of the Effectiveness of Interventions to Prevent and Respond to Violence against Persons with Disabilities. J Interpers Violence 2014; 29: 3207-3226

Muccigrosso L. Sexual Abuse Prevention Strategies and Programs for Persons with Developmental Disabilities. Sex Disabil 1991; 9: 261-271

${ }^{*}$ Mueller-Johnson K, Eisner MP, Obsuth I. Sexual Victimization of Youth with a Physical Disability: An Examination of Prevalence Rates and Risk and Protective Factors. J Interpers Violence 2014; 29: 3180-3206

Noack C, Schmid HJ. Sexuelle Gewalt gegen Menschen mit geistiger Behinderung. Eine verleugnete Realität. Ergebnisse und Fakten einer bundesweiten Befragung. Esslingen: Lebenshilfe 1994

*Normand CL, Sallafranque-St-Louis F. Cybervictimization of Young People with an Intellectual or Developmental Disability: Risks Specific to Sexual Solicitation. J Appl Res Int Disabil 2016; 29: 99-110

O’Day B. Preventing Sexual Abuse of Persons with Disabilities: A Curriculum for Hearing Impaired, Physically Disabled, Blind and Mentally Retarded Students. Santa Cruz: Network Publications 1983

* Ohlsson Gotby V, Lichtenstein P, Långström N, Pettersson E. Childhood Neurodevelopmental Disorders and Risk of Coercive Sexual Victimization in Childhood and Adolescence - a Population-Based Prospective Twin Study. J Child Psychol Psychiatry 2018; 59: 957-965

${ }^{*}$ Reid JA. Sex Trafficking of Girls with Intellectual Disabilities: An Exploratory Mixed Methods Study. Sex Abuse 2018; 30: 107-131

Reis O, Häßler F, Daubmann A, Chodan W. Learned Behavior Hardly Translates to Reality - a Randomized Controlled Trial on Sexual Abuse Prevention for Girls with Intellectual Disabilities; eingereicht zur Publikation

*Schenkel LS, Rothman-Marshall G, Schlehofer DA, Towne TL, Burnash DL, Priddy BM. Child Maltreatment and Trauma Exposure among Deaf and Hard of Hearing Young Adults. Child Abuse Negl 2014; 38: 1581-1589

Seligmann S. Sexueller Missbrauch von Kindern. Ansätze einer Prävention für die Sonderschulpädagogik. Hamburg: Kovac 1996

Senn CY. Gegen jedes Recht. Sexueller Missbrauch und geistige Behinderung. Berlin: Donna Vita 1993

Sequeira H, Hollins S. Clinical Effects of Sexual Abuse on People with Learning Disability. Br J Psychiatry 2003; 182: 13-19

Singer N. Evaluation of a Self-Protection Group for Clients Living in a Residential Group Home. Br J Dev Disabil 1996; 82: 54-62

Sobsey D, Mansell S. Sexual Abuse Patterns of Children with Disabilities. IJ Child Rights 1994; 2: 96-100

Sullivan PM, Knutson JF. Maltreatment and Disabilities: A Population-Based Epidemiological Study. Child Abuse Negl 2000; 24: 1257-1273

Stinkes U. Sexualität und Behinderung - kein Tabuthema mehr?! Stuttgart: Margarete-Steiff-Schule Stuttgart 2006 [Als Online-Dokument: https:// www.margarete-steiff-schule.de/application/files/2015/0446/7138/ vortrag_stinkes.pdf]

*Stone M. Preventing Sexual Violence against People with Disabilities: Empowerment Self-Defense, Risk Reduction Education and Organizational Change. In: Orchowski L, Gidycz C, Hrsg. Sexual Assault Risk Reduction and Resistance. London, UK, San Diego, CA, Cambridge, MA: Academic Press 2018; 353-378

Tang CS, Lee YK. Knowledge on Sexual Abuse and Self-Protection Skills: A Study on Female Chinese Adolescents with Mild Mental Retardation. Child Abuse Negl 1999; 23: 269-279

Tharinger D, Horton CB, Millea S. Sexual Abuse and Exploitation of Children and Adults with Mental Retardation and Other Handicaps. Child Abuse Negl 1990; 14: 301-312

*Thomas N, Nattala P, Seshadri S. Effectiveness of Behavioral Skills Training (BST) on Knowledge of Sexual Abuse and Resistance Ability among 
Children with Intellectual Disability: A Pilot Study. Int J Child Dev Ment Health 2018; 6: 22-30

Urbann K. Konzeption und Evaluation des evidenzbasierten Präventionstrainings „STARK mit SAM“ für Schülerinnen und Schüler mit Hörbehinderung. Dissertation, Technische Universität Dortmund 2018 [Als Online-Dokument: https://d-nb.info/1176189662/34]

*Urbann K, Bienstein P, Kaul T. The Evidence-Based Sexual Abuse Prevention Program: Strong With Sam. J Deaf Stud Deaf Educ 2020; 25: 421-429

*Vara A, Quintana JM, Escorial S, Manzanero AL. Descriptive Analysis of the Characteristics of Proven Cases of Sexual Abuse in Victims with Intellectual Disabilities and Children with Typical Development in Spain. J Interpers Violence 2019; doi:10.1177/0886260519888201

*Warraitch A, Amin R, Rashid A. Evaluation of a School-Based Sexual Abuse Prevention Program for Female Children with Intellectual Disabilities in Rural Pakistan - a Feasibility Study. Appl Nurs Res 2021; 57: 151391

Watson M, Bain A, Houghton S. A Preliminary Study in Teaching Self-Protective Skills to Children with Moderate and Severe Mental Retardation. J Spec Educ 1992; 26: 181-194

[WHO] World Health Organization. International Classification of Functioning, Disability, and Health: Children \& Youth Version. Genf: WHO 2007

*Willott S, Badger W, Evans V. People with an Intellectual Disability: UnderReporting Sexual Violence. J Adult Prot 2020; 22: 75-88

*Wissink IB, van Vugt E, Moonen X, Stams GJ], Hendriks J. Sexual Abuse Involving Children with an Intellectual Disability (ID): A Narrative Review. Res Dev Disabil 2015; 36: 20-35

*Wissink IB, van Vugt E, Smits IA, Moonen XM, Stams GJJ. Reports of Sexual Abuse of Children in State Care: A Comparison between Children with and without Intellectual Disability. J Intellect Dev Disabil 2018; 43: 152-163

Yeater EA, O’Donohue W. Sexual Assault Prevention Programs: Current Issues, Future Directions and the Potential Efficacy of Interventions with Women. Clin Psychol Rev 1999; $19: 739-771$ 